\title{
HIERARCHICAL MULTILEVEL OPTIMIZATION WITH MULTIPLE-LEADERS MULTIPLE-FOLLOWERS SETTING AND NONSEPARABLE OBJECTIVES
}

\author{
Addis Belete Zewde ${ }^{1}$ And Semu Mitiku Kassa ${ }^{2, *}$ (1)
}

\begin{abstract}
Hierarchical multilevel multi-leader multi-follower problems are non-cooperative decision problems in which multiple decision-makers of equal status in the upper-level and multiple decisionmakers of equal status are involved at each of the lower-levels of the hierarchy. Much of solution methods proposed so far on the topic are either model specific which may work only for a particular sub-class of problems or are based on some strong assumptions and only for two level cases. In this paper, we have considered hierarchical multilevel multi-leader multi-follower problems in which the objective functions contain separable and non-separable terms (but the non-separable terms can be written as a factor of two functions, a function which depends on other level decision variables and a function which is common to all objectives across the same level) and shared constraint. We have proposed a solution algorithm to such problems by equivalent reformulation as a hierarchical multilevel problem involving single decision maker at all levels of the hierarchy. Then, we applied a multi-parametric algorithm to solve the resulting single leader single followers problem.
\end{abstract}

Mathematics Subject Classification. 91A65, 91A06, 91A10, 90C26.

Received November 1, 2020. Accepted September 18, 2021.

\section{INTRODUCTION}

Multilevel multi-leader multi-follower (MLMF) game is a non-cooperative decision system in which there are multiple higher-level decision-makers (who are referred to as leaders) and many lower-level decision-makers (who are referred to as followers). In this formulation, the role of leaders and followers may not refer to the level of management hierarchy, rather it indicates the sequence of actions in the decision process among themselves. The leaders compete in a Nash game constrained by the equilibrium conditions of another Nash game among the followers. In the sequential part of the game Stackelberg behavior is assumed, from which the leaders make their decision first by competing in a Nash game constrained by the equilibrium conditions of another Nash game among the followers and the followers react by optimizing their objective functions conditioned on the leaders' decision. Since originally appeared in a 1973 paper by Bracken and McGill [1] multilevel decision-making (some authors designated it as multilevel programming or multilevel optimization) often appears in many decentralized

\footnotetext{
Keywords. Hierarchical game, multi-leader multi-follower, Stackelberg game, Nash game, equivalent reformulation, multiparametric algorithm.

1 Department of Mathematics, Addis Ababa University, P.O.Box 1176, Addis Ababa, Ethiopia.

2 Department of Mathematics and Statistical Sciences, Botswana International University of Science and Technology, P/Bag 16, Palapye, Botswana.

* Corresponding author: kassas@biust.ac.bw
} 
management problems in the real world and has motivated a number of researches on decision models, solution approaches and applications.

One of the solution approaches in solving multilevel-MLMF games uses a reformulation that replaces the lower-level problems by their optimality conditions which results in a mathematical problem classified as an equilibrium problem with equilibrium constraints (EPECs). Although there are several EPECs which have been shown to admit an equilibrium, there are also fairly simple EPECs which admit no equilibrium as shown in [18]. Under this approach, Okuguchi [17] presented models for multi-leader-follower games in a Cournot regime. With the assumption that each leader can exactly anticipate the aggregate reaction curve of the followers, Okuguchi proved existence of an equilibrium solution for such problems. Moreover, uniqueness of such a solution is asserted for a special case of the problem where all leaders share an identical cost function and make identical decisions. Sherali [21] also studied a multi-leader-follower game, referring it as a Stackelberg model, by associating it with EPECs. In forward market equilibrium model, Su [22] extended the existence result of [17] and [21] under some weaker conditions. The works of Pang and Fukushima [19] and Leyffer and Munson [16] are also categorized under this reformulation.

Replacing the lower-level problems by their optimality conditions requires convexity of the problems and some strict regularity conditions on the constraints. Under such condition it can be shown that the reformulated problem is equivalent to the original one. However, if the level of decision hierarchy is more than two then the equilibrium problem of the lower levels become highly non-convex and it will be difficult to establish continuity of the set-valued solution (equilibrium) function. Moreover, for problems with more than three hierarchical levels with multiple leaders and multiple followers at each level, it is not possible also to apply the principle of variational inequality to solve them.

An alternative solution approach in solving multilevel-MLMF problems is reformulation of the problem as an equivalent hierarchical multilevel problem involving a single decision maker at all levels of the hierarchy. In such a case, once it is equivalently reformulated as a hierarchical multilevel problem, one can apply existing solution approaches for multilevel optimization models to solve the problem, though such methods themselves are limited. Wang et al. [24] used this reformulation for a convex bilevel problem with multiple followers and separable second-level objective functions. Under some weaker conditions, this reformulation is also employed in the works of Kulkarni and Shanbhag [15], Kassa and Kassa [14], and Kassa [12]. The type of multilevelMLMF games considered in [15] is a problem in which the objective function of each player consists of separable terms and common non-separable terms across all the followers. Extending this result, Kassa and Kassa [14] reformulated a class of multilevel single-leader multiple-follower games, that consist of separable terms and nonseparable terms across all the followers parameterized by constant positive weights. Motivated by this latest method, the authors have proposed in $[25,26]$ an equivalent reformulation procedure to solve multiple-leaders multiple-followers problems with any finite level of hierarchy. However, this procedure increases the level of the hierarchy by one level and requires all the assumption in [14] to be satisfied.

Recently, multilevel-MLMF decision problems have been increasingly appearing in decentralized management situations in the current age of integrated economic developments where business firms work in a decentralized manner in a complex commercial networks comprised of suppliers, manufacturers, sales and logistics companies, customers and other specialized service functions [8]. Therefore, this paper considers a hierarchical multi-leader multi-follower problems in which multiple leaders of equal status in the upper-level and multiple followers of equal status are involved at each lower-level of the hierarchy. We assume that the objective at all levels have separable and non-separable terms (but the non-separable terms can be written as a factor of two functions where the first one is a function of other level decision variables and the second factor is common to all objectives across the same level), at each level there is a shared constraint common to all problems of same level, the leader and followers have their own decision variables and objective functions, and the leader can only influence the reactions of followers through their decision variables, while the followers optimize their objective functions in view of the decisions of the leader and other middle level decision agents. Due to its computational difficulties and mathematical complexities such as non-convexity and NP-hardness such optimization problems are lacking 
efficient algorithms. The main objective of this paper is to propose a solution approach for a more general class of multilevel MLMF problems.

The remainder of the paper is organized into six sections. The next section presents the mathematical formulation of general multilevel-MLMF games. Section 3 describes the proposed equivalent reformulations of bilevel-MLMF problems into a hierarchical bilevel problem involving a single decision maker at each level of the hierarchy. In Section 4, a multi-parametric programming problems are described. Section 5 presents an algorithm to solve multilevel-MLMF problems using equivalent reformulation and multi-parametric approach. Numerical examples are provided in Section 6 to illustrate the procedures of the algorithms and the paper ends with concluding remarks in Section 7.

\section{General formulations of MUltileVEL-MLMF GAMES}

Consider a $k$-level hierarchical multi-leader multi-follower game involving $N_{1}$ decision makers at the first-level, $N_{2}$ decision makers at the second-level, $\ldots$, and $N_{k}$ decision makers at the $k^{t h}$-level. Under the assumptions that (i) there is a shared constraint common to all problems at the same level, (ii) the reaction of all followers are consistent across all the leaders at all hierarchical levels, and (iii) leaders and followers at all levels have their own decision variables, objective functions and constraints, the problem can be formulated mathematically as follows.

For $n=1, \ldots, N_{1}$, the vector $\left(y_{1}^{n}, y_{2}, \ldots, y_{k}\right)$ solves an optimization problem,

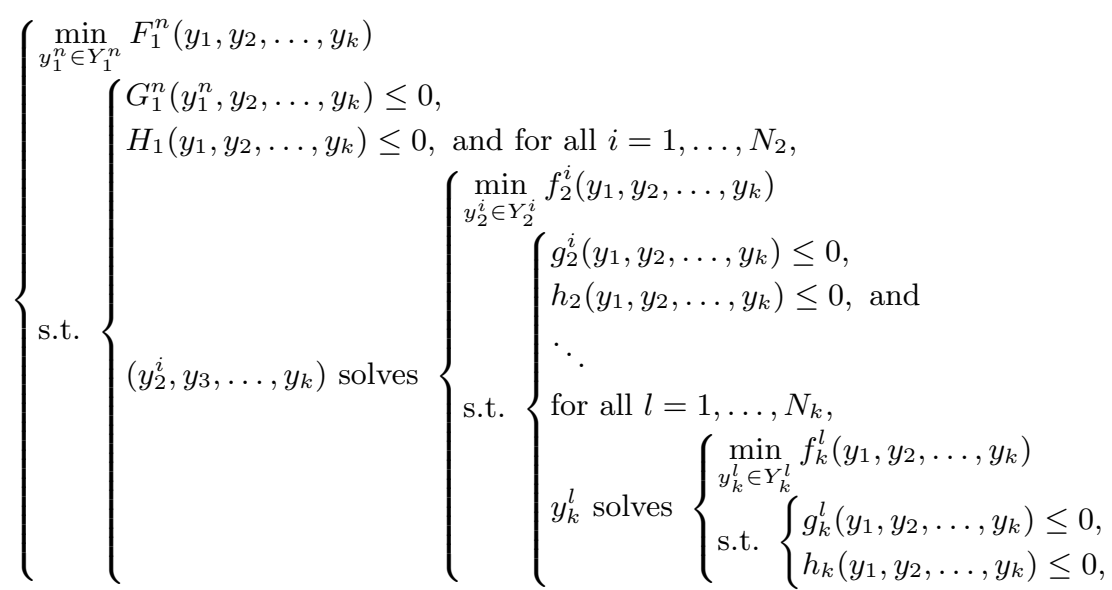

where $y_{1}=\left(y_{1}^{1}, \ldots, y_{1}^{N_{1}}\right)$ is a decision vector for the leaders' optimization and $(\forall n) \quad y_{1}^{n} \in Y_{1}^{n}$ is a decision vector of the $n^{t h}$-leader and $y_{1}^{-n}=\left(y_{1}^{1}, \ldots, y_{1}^{n-1}, y_{1}^{n+1}, \ldots, y_{1}^{N_{1}}\right)$ is a vector of the decision variables for all leaders without $y_{1}^{n}$; note that as customary we may write $y_{1}=\left(y_{1}^{n} ; y_{1}^{-n}\right)$. The shared constraint $H_{1}$ is common to all leaders whereas, the constraint $G_{1}^{n}$ is only for the $n$ th-leader. Similarly, at the second-level, $y_{2}=\left(y_{2}^{1}, \ldots, y_{2}^{N_{2}}\right)$ is a decision vector for the second-level problem and $\forall i \in\left\{1,2, \ldots, N_{2}\right\}, y_{2}^{i} \in Y_{2}^{i}$ is a decision vector of the $i$ th-player at the second-level optimization problem and $y_{2}^{-i}=\left(y_{2}^{1}, \ldots, y_{2}^{i-1}, y_{2}^{i+1}, \ldots, y_{2}^{N_{2}}\right)$ is a vector of the decision variables of all second-level players without the vector $y_{2}^{i}$; and $y_{2}=\left(y_{2}^{i} ; y_{2}^{-i}\right)$. The shared constraint $h_{2}$ is common to all second-level players whereas, the constraint $G_{2}^{i}$ affects only the $i$ th player of the second-level problem.

Continuing the same way as above, finally, at $k$ th-level, $y_{k}=\left(y_{k}^{1}, \ldots, y_{k}^{N_{k}}\right)$ is a decision vector for the $k^{t h}$-level problem and $\forall l \in\left\{1,2, \ldots, N_{k}\right\}, y_{k}^{l} \in Y_{k}^{l}$ is a decision vector of the $l$ th-player at the $k$ th-level problem and $y_{k}^{-l}=\left(y_{k}^{1}, \ldots, y_{k}^{l-1}, y_{k}^{l+1}, \ldots, y_{k}^{N_{k}}\right)$ is a vector of the decision variables of all $k$ th-level players without the vector $y_{k}^{l}$; and $y_{k}=\left(y_{k}^{l} ; y_{k}^{-l}\right)$. The shared constraint $h_{k}$ is common to all $k$ th-level players whereas, the constraint $G_{k}^{l}$ is assumed to affect only the $l$ th-player of the $k$ th-level problem.

In problem (2.1), if $k=2$ the problem is called a bilevel-MLMF game. As a bilevel problem, all leaders in the upper-level compete with each other in a non-cooperative Nash game and make their decisions first 
by anticipating the responses of all the followers. Upon receipt of the leaders' decisions, all followers compete with each other in a parametric non-cooperative Nash game in the lower level with the strategies of leaders as exogenous parameters [10]. This makes the problem of solving a multilevel problem having multiple decision makers at each decision level quite difficult and complex, particularly when there is information exchange between followers. In many applications, for instance in supply chain management models, however there are more than two decision agents, like manufacturers, distributers, retailers (or vendors), etc. If we follow the traditional approach, there will appear non-convex terms and shared variables across the followers at the $(k-$ 1)th-level due to complementarity conditions from the $k$ th-level followers. This may result in challenging task in the process of solving multilevel-MLMF problem having shared resources and information.

At each levels of decision hierarchy in multilevel-MLMF problems, one need to solve parametric generalized Nash Equilibrium problems, where the variables from upper levels are considered as parameters. It is well known that solving such problems can be a tedious and error-prone task [23]. Many of the solution approaches for solving problems with MLMF nature apply a reformulation of the Nash equilibrium problem and Stackelberg equilibrium problem as an 'equivalent' variational inequality (VI) problems and mathematical problems with equilibrium constraints (MPECs), respectively. But such reformulations have several limitations as mentioned in [16]. In addition, a standard approach in MPECs requires ascertaining when the reaction map admits fixed points. But this is difficult due to the lack of continuity in the solution set associated with the equilibrium constraints capturing the follower equilibrium. Due to these challenges most of the solution methods work only for particular subclasses of multilevel-MLMF games which satisfy some strict conditions (such as, strict convexity, separability, etc.) for two levels. In the next section, we present a reformulation of a class of multiple leaders and multiple followers problems into an equivalent bilevel problem involving only a single decision maker at both levels of the hierarchy, which can be extended later to any finite hierarchical levels.

\section{Equivalent ReFormulation of BileVEL-MLMF GAMES}

Consider the following bilevel-MLMF game in which $N$ leaders compete in a non-cooperative game subject to the equilibrium conditions of $M$ followers competing in a lower-level game given leaders' level decisions. If we denote $x^{i}, i \in\{1, \ldots, N\}$, the decision variables vector for leader $i$ and $y^{j}, j \in\{1, \ldots, M\}$, the decision variables for follower $j$. The leaders' and followers' variables are abbreviated, respectively by $x=\left(x^{1}, \ldots, x^{N}\right)$ and $y=\left(y^{1}, \ldots, y^{M}\right)$. For each of the leaders, $i=1, \ldots, N$, the Stackelberg game played by leader $i$ is given by:

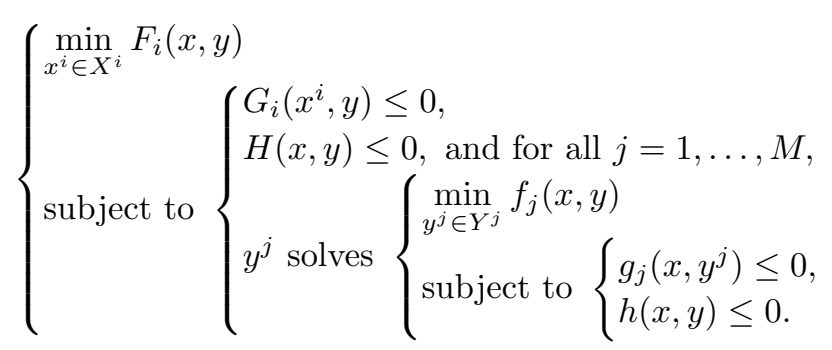

Let us assume that, for all $i$ and for each $j$, the functions $F_{i}, G_{i}, H, h, f_{j}, g_{j}$ in (3.1) are twice continuously differentiable, and that the followers' constraint functions satisfy the Guignard constraint qualifications conditions [7]. Let us define the following sets that can be used in the preceding sections to characterize solutions of the problem (3.1).

(i) The feasible set of problem (3.1) is given by

$$
\mathcal{F}=\left\{(x, y): H(x, y) \leq 0, h(x, y) \leq 0,(\forall i) G_{i}\left(x^{i}, y\right) \leq 0,(\forall j) g_{j}\left(x, y^{j}\right) \leq 0\right\} .
$$

(ii) For any given leaders' strategy $x$, the feasible set for the $j^{\text {th }}$-follower is defined as

$$
\mathcal{F}_{j}\left(x, y^{-j}\right)=\left\{y^{j} \in Y^{j}: g_{j}\left(x, y^{j}\right) \leq 0, h(x, y) \leq 0\right\} .
$$


(iii) The Nash rational reaction set for the $j$ th-follower, is defined by,

$$
\mathcal{R}_{j}\left(x, y^{-j}\right)=\left\{\bar{y}^{j} \in Y^{j}: \bar{y}^{j} \in \underset{y^{j}}{\operatorname{argmin}}\left\{f_{j}(x, y) \text { subject to } y^{j} \in \mathcal{F}_{j}\left(x, y^{-j}\right)\right\}\right\} .
$$

(iv) The feasible set for the $i$ th-leader, is defined as

$$
\mathcal{F}_{i}\left(x^{-i}\right)=\left\{\left(x^{i}, y\right):(x, y) \in \mathcal{F},(\forall j) y^{j} \in \mathcal{R}_{j}\left(x, y^{-j}\right)\right\} .
$$

(v) The Nash rational reaction set for the $i$ th-leader is given by

$$
\mathcal{R}_{i}\left(x^{-i}\right)=\left\{\left(\bar{x}^{i}, y\right): \bar{x}^{i} \in \underset{x^{i}}{\operatorname{argmin}}\left\{F_{i}(x, y) \text { subject to }\left(x^{i}, y\right) \in \mathcal{F}_{i}\left(x^{-i}\right)\right\}\right\} .
$$

(vi) The set of Stackelberg-Nash equilibrium points of problem (3.1) is given by

$$
\mathcal{E}=\left\{(x, y) \in \mathcal{F}:(\forall i)\left(x^{i}, y\right) \in \mathcal{R}_{i}\left(x^{-i}\right),(\forall j) y^{j} \in \mathcal{R}_{j}\left(x, y^{*,-j}\right)\right\} .
$$

Remark 3.1. If $\left(x^{*}, y^{*}\right) \in \mathcal{E}$, for $\forall(x, y) \in \mathcal{F},(\forall i)$ and $(\forall j)$ we have

$$
F_{i}\left(x^{*}, y^{*}\right) \leq F_{i}\left(x, y^{*}\right) \leq F_{i}(x, y) \text { and } f_{j}\left(x^{*}, y^{*}\right) \leq f_{j}\left(x^{*}, y\right) \leq f_{j}(x, y) .
$$

Using the reaction sets, the leaders Nash problem of (3.1) is given by

$$
\forall i\left(x^{i}, y\right) \in\left\{\begin{array}{l}
\underset{x^{i}}{\operatorname{argmin}} F_{i}(x, y) \\
\text { subject to }\left(x^{i}, y\right) \in \mathcal{R}_{i}\left(x^{-i}\right) .
\end{array}\right.
$$

Definition 3.2. A tuple $\left(x^{*}, y\right)=\left(x^{*, i}, x^{*,-i}, y\right)$ is called an optimal Nash equilibrium solution to the problem (3.2), if it satisfies the following conditions:

$$
F_{i}\left(x^{*, i}, x^{*,-i}, y\right) \leq F_{i}\left(x^{i}, x^{*,-i}, y\right),(\forall i)\left(x^{i}, y\right) \in \mathcal{R}_{i}\left(x^{*,-i}\right) .
$$

Given a strategy $x$ of the leaders, each of the $M$ followers play the Nash game:

$$
(\forall j) y^{*, j} \in\left\{\begin{array}{l}
\underset{y^{j}}{\operatorname{argmin}} f_{j}(x, y) \\
\text { subject to } y^{j} \in \mathcal{R}_{j}\left(x, y^{-j}\right)
\end{array}\right.
$$

Definition 3.3. A tuple $y^{*}=\left(y^{*, j}, y^{*,-j}\right)$ is called an optimal Nash equilibrium solution to the problem (3.3), if it satisfies the following conditions:

$$
f_{j}\left(x, y^{*, j}, y^{*,-j}\right) \leq f_{j}\left(x, y^{j}, y^{*,-j}\right),(\forall j) y^{j} \in \mathcal{R}_{j}\left(x, y^{*,-j}\right) .
$$

When the Nash problem (3.2) has objective functions with separable terms and a non-separable term which is common to all leaders $\left(i . e\right.$. , for each $i$ if the objective functions are written as $F_{i}\left(x^{i}, x^{-i}, y\right)=\hat{F}_{i}\left(x^{i}\right)+\check{F}_{i}\left(x^{-i}\right)+$ $\tilde{F}(x, y))$, by defining a quasi-potential function

$$
\mathbf{F}(x, y)=\left[\sum_{i=1}^{N} \hat{F}_{i}\left(x^{i}\right)\right]+\tilde{F}(x, y)
$$


Kulkarni and Shanbhag [15] equivalently reformulated problem (3.2) as a single optimization problem

$$
\begin{aligned}
& \min _{x} \mathbf{F}(x, y)=\left[\sum_{i=1}^{N} \hat{F}_{i}\left(x^{i}\right)\right]+\tilde{F}(x, y) \\
& \text { subject to }\left(x^{i}, y\right) \in \mathcal{R}_{i}\left(x^{-i}\right)(\forall i) .
\end{aligned}
$$

And they have shown that, the global minimizers of problem (3.4) are global equilibria of the problem (3.2). In general, it has been shown in [15] that if the objectives of the leaders and the followers admit a quasi-potential function formulation, then the global minimizers of this quasi-potential function problem are global equilibria of the MLMF game.

The quasi-potential function problem of Kulkarni and Shanbhag considered in [15] covers only a class of games whose objective functions are written as separable terms and a non-separable term which is common to all decision makers at that level. In this work we have considered a game in which the objective functions contain a separable and non-separable terms; but the non-separable terms can be written as a factor of two functions where the first one is a function of other level decision variables and the second factor is common to all objectives across the same level.

We make the following assumptions on the structure of the objective functions of the multi-leader multifollower game that will enable us to reformulate a bilevel-MLMF game as a hierarchical bilevel game involving a single decision maker at both levels:

(A1) The leaders objective can be written as

$$
F_{i}(x, y)=\hat{F}_{i}\left(x^{i}, y\right)+\bar{F}_{i}\left(x^{-i}, y\right)+\rho_{i}(y) \tilde{F}(x, y)=\breve{F}_{i}\left(x^{i}, x^{-i}, y\right)+\rho_{i}(y) \tilde{F}(x, y),
$$

where, for any $y \in Y,(\forall i)\left(0<\rho_{i}(y)<\infty\right)$.

(A2) The followers objective can be written as

$$
f_{j}(x, y)=\hat{f}_{j}\left(x, y^{j}\right)+\bar{f}_{j}\left(x, y^{-j}\right)+\delta_{j}(x) \tilde{f}(x, y)=\breve{f}_{j}\left(x, y^{j}, y^{-j}\right)+\delta_{j}(x) \tilde{f}(x, y)
$$

where, for any $x \in X,(\forall j)\left(0<\delta_{j}(x)<\infty\right)$.

(A3) $\rho_{i}(\cdot)$ and $\delta_{j}(\cdot)$ are twice continuously differentiable functions and uniformly bounded away from zero.

Lemma 3.4. If assumptions (A1) and (A2) hold for any $(x, y) \in X \times Y$, then there exist functions $\pi_{F}(x, y)$ and $\pi_{f}(x, y)$ such that

$$
\begin{aligned}
& \breve{F}_{i}\left(x^{*, i}, x^{*,-i}, y\right)-\breve{F}_{i}\left(x^{i}, x^{*,-i}, y\right)=\rho_{i}(y)\left[\pi_{F}\left(x^{*, i}, x^{*,-i}, y\right)-\pi_{F}\left(x^{i}, x^{*,-i}, y\right)\right], \quad(\forall i), \\
& \breve{f}_{j}\left(x, y^{*, j}, y^{*,-j}\right)-\breve{f}_{j}\left(x, y^{j}, y^{*,-j}\right)=\delta_{j}(x)\left[\pi_{f}\left(x, y^{*, j}, y^{*,-j}\right)-\pi_{f}\left(x, y^{j}, y^{*,-j}\right)\right], \quad(\forall j) .
\end{aligned}
$$

Proof. For $(x, y) \in X \times Y$ and $0<\rho_{i}(y)<\infty$, define the function

$$
\pi_{F}(x, y)=\sum_{i=1}^{N} \frac{1}{\rho_{i}(y)} \hat{F}_{i}\left(x^{i}, y\right) .
$$

Note that, because of Assumption (A1), the function $\pi_{F}$ is well defined. Then, for any $i$ we have 


$$
\begin{aligned}
\rho_{i}(y)[ & \left.\pi_{F}\left(x^{*, i}, x^{*,-i}, y\right)-\pi_{F}\left(x^{i}, x^{*,-i}, y\right)\right] \\
= & \rho_{i}(y)\left[\sum_{k=1, k \neq i}^{N} \frac{1}{\rho_{k}(y)} \hat{F}_{k}\left(x^{*, k}, y\right)-\sum_{k=1, k \neq i}^{N} \frac{1}{\rho_{k}(y)} \hat{F}_{k}\left(x^{*, k}, y\right)\right] \\
& \quad+\rho_{i}(y)\left[\frac{1}{\rho_{i}(y)} \hat{F}_{i}\left(x^{*, i}, y\right)-\frac{1}{\rho_{i}(y)} \hat{F}_{i}\left(x^{i}, y\right)\right] \\
= & \rho_{i}(y)\left[\frac{1}{\rho_{i}(y)} \hat{F}_{i}\left(x^{*, i}, y\right)-\frac{1}{\rho_{i}(y)} \hat{F}_{i}\left(x^{i}, y\right)\right] \\
= & \hat{F}_{i}\left(x^{*, i}, y\right)-\hat{F}_{i}\left(x^{i}, y\right) \\
= & {\left[\hat{F}_{i}\left(x^{*, i}, y\right)+\bar{F}_{i}\left(x^{*,-i}, y\right)\right]-\left[\hat{F}_{i}\left(x^{i}, y\right)+\bar{F}_{i}\left(x^{*,-i}, y\right)\right] } \\
= & \breve{F}_{i}\left(x^{*, i}, x^{*,-i}, y\right)-\breve{F}_{i}\left(x^{i}, x^{*,-i}, y\right) .
\end{aligned}
$$

Implying that

$$
\breve{F}_{i}\left(x^{*, i}, x^{*,-i}, y\right)-\breve{F}_{i}\left(x^{i}, x^{*,-i}, y\right)=\rho_{i}(y)\left[\pi_{F}\left(x^{*, i}, x^{*,-i}, y\right)-\pi_{F}\left(x^{i}, x^{*,-i}, y\right)\right],
$$

Similarly, for $(x, y) \in X \times Y$ and $0<\delta_{i}(x)<\infty$ by defining

$$
\pi_{f}(x, y)=\sum_{j=1}^{M} \frac{1}{\delta_{j}(x)} \hat{f}_{j}\left(x, y^{j}\right)
$$

we will have

$$
\breve{f}_{j}\left(x, y^{*, j}, y^{*,-j}\right)-\breve{f}_{j}\left(x, y^{j}, y^{*,-j}\right)=\delta_{j}(x)\left[\pi_{f}\left(x, y^{*, j}, y^{*,-j}\right)-\pi_{f}\left(x, y^{j}, y^{*,-j}\right)\right],(\forall j) .
$$

Hence, the conclusion of the Lemma follows.

For a given strategy $y$, define the following optimization problem,

$$
\left\{\begin{array}{l}
\min _{x} \mathbf{F}(x, y)=\left[\sum_{i=1}^{N} \frac{1}{\rho_{i}(y)} \hat{F}_{i}\left(x^{i}, y\right)\right]+\tilde{F}(x, y) \\
\text { subject to }\left(x^{i}, y\right) \in \mathcal{R}_{i}\left(x^{-i}\right)(\forall i)
\end{array}\right.
$$

Equivalence between optimal solution of (3.5) and equilibrium point of the Nash problem (3.2) can be established based on the following theorem.

Theorem 3.5. Suppose that problem (3.2) satisfy Assumption (A1). If ( $\left.x^{*}, y\right)$ is a global optimal solution of (3.5), then $\left(x^{*}, y\right)$ is a global Nash equilibrium point of (3.2).

Proof. Let $\left(x^{*}, y\right)=\left(x^{*, i}, x^{*,-i}, y\right)$ be an optimal solution to $(3.5)$, then

$$
\sum_{i=1}^{N} \frac{1}{\rho_{i}(y)} \hat{F}_{i}\left(x^{*, i}, y\right)+\tilde{F}\left(x^{*, i}, x^{*,-i}, y\right) \leq \sum_{i=1}^{N} \frac{1}{\rho_{i}(y)} \hat{F}_{i}\left(x^{i}, y\right)+\tilde{F}\left(x^{i}, x^{-i}, y\right), \forall\left(x^{i}, y\right) \in \mathcal{R}_{i}\left(x^{-i}\right) .
$$

If we let $\pi_{F}(x, y)=\sum_{i=1}^{N} \frac{1}{\rho_{i}(y)} \hat{F}_{i}\left(x^{i}, y\right)$, it will follow that

$$
\pi_{F}\left(x^{*, i}, x^{*,-i}, y\right)+\tilde{F}\left(x^{*, i}, x^{*,-i}, y\right) \leq \pi_{F}\left(x^{i}, x^{-i}, y\right)+\tilde{F}\left(x^{i}, x^{-i}, y\right), \forall\left(x^{i}, y\right) \in \mathcal{R}_{i}\left(x^{-i}\right) .
$$


Particularly for $x^{-i}=x^{*,-i}$ and $\forall\left(x^{i}, y\right) \in \mathcal{R}_{i}\left(x^{*,-i}\right)$, we have

$$
\pi_{F}\left(x^{*, i}, x^{*,-i}, y\right)+\tilde{F}\left(x^{*, i}, x^{*,-i}, y\right) \leq \pi_{F}\left(x^{i}, x^{*,-i}, y\right)+\tilde{F}\left(x^{i}, x^{*,-i}, y\right) .
$$

By rearranging the above expression, we have

$$
\pi_{F}\left(x^{*, i}, x^{*,-i}, y\right)-\pi_{F}\left(x^{i}, x^{*,-i}, y\right)+\tilde{F}\left(x^{*, i}, x^{*,-i}, y\right) \leq \tilde{F}\left(x^{i}, x^{*,-i}, y\right) .
$$

Multiplying both sides of the last inequality by $\rho_{i}(y)>0$, results in $\forall\left(x^{i}, y\right) \in \mathcal{R}_{i}\left(x^{*,-i}\right)$

$$
\rho_{i}(y)\left[\pi_{F}\left(x^{*, i}, x^{*,-i}, y\right)-\pi_{F}\left(x^{i}, x^{*,-i}, y\right)\right]+\rho_{i}(y) \tilde{F}\left(x^{*, i}, x^{*,-i}, y\right) \leq \rho_{i}(y) \tilde{F}\left(x^{i}, x^{*,-i}, y\right) .
$$

Using the results from Lemma 3.4,

$$
\rho_{i}(y)\left[\pi_{F}\left(x^{*, i}, x^{*,-i}, y\right)-\pi_{F}\left(x^{i}, x^{*,-i}, y\right)\right]=\breve{F}_{i}\left(x^{*, i}, x^{*,-i}, y\right)-\breve{F}_{i}\left(x^{i}, x^{*,-i}, y\right) .
$$

That means, in the above inequality, $\forall\left(x^{i}, y\right) \in \mathcal{R}_{i}\left(x^{*,-i}\right)$ we have

$$
\breve{F}_{i}\left(x^{*, i}, x^{*,-i}, y\right)-\breve{F}_{i}\left(x^{i}, x^{*,-i}, y\right)+\rho_{i}(y) \tilde{F}\left(x^{*, i}, x^{*,-i}, y\right) \leq \rho_{i}(y) \tilde{F}\left(x^{i}, x^{*,-i}, y\right) .
$$

Implying, $\forall\left(x^{i}, y\right) \in \mathcal{R}_{i}\left(x^{*,-i}\right)$

$$
\breve{F}_{i}\left(x^{*, i}, x^{*,-i}, y\right)+\rho_{i}(y) \tilde{F}\left(x^{*, i}, x^{*,-i} y\right) \leq \breve{F}_{i}\left(x^{i}, x^{*,-i}, y\right)+\rho_{i}(y) \tilde{F}\left(x^{i}, x^{*,-i}, y\right) .
$$

This is equivalent to

$$
F_{i}\left(x^{*, i}, x^{*,-i}, y\right) \leq F_{i}\left(x^{i}, x^{*,-i}, y\right),(\forall i)\left(x^{i}, y\right) \in \mathcal{R}_{i}\left(x^{*,-i}\right) .
$$

From inequality (3.6) we can see that the tuple $\left(x^{*}, y\right)$, satisfies Definition 3.2. Therefore, $\left(x^{*}, y\right)$ is a global Nash equilibrium point of (3.2).

For a given strategy $x$, define the following optimization problem,

$$
\left\{\begin{array}{l}
\min _{y} \mathbf{f}(x, y)=\left[\sum_{j=1}^{M} \frac{1}{\delta_{j}(x)} \hat{f}_{j}\left(x, y^{j}\right)\right]+\tilde{f}(x, y) \\
\text { subject to } y^{j} \in \mathcal{R}_{j}\left(x, y^{-j}\right)(\forall j) .
\end{array}\right.
$$

Then the relation between (3.7) and (3.3) can be established as follows.

Theorem 3.6. Suppose that problem (3.3) satisfies Assumption (A2). If $y^{*}$ is an optimal solution of (3.7), then $y^{*}$ is a Nash reaction point of (3.3).

Proof. Let $y^{*}=\left(y^{*, j}, y^{*,-j}\right)$ be an optimal solution for (3.7), then

$$
\sum_{j=1}^{M} \frac{1}{\delta_{j}(x)} \hat{f}_{j}\left(x, y^{*, j}\right)+\tilde{f}\left(x, y^{*, j}, y^{*,-j}\right) \leq \sum_{j=1}^{M} \frac{1}{\delta_{j}(x)} \hat{f}_{j}\left(x, y^{j}\right)+\tilde{f}\left(x, y^{j}, y^{-j}\right), \forall y^{j} \in \mathcal{R}_{j}\left(x, y^{-j}\right) .
$$

If we let $\pi_{f}(x, y)=\sum_{j=1}^{M} \frac{1}{\delta_{j}(x)} \hat{f}_{j}\left(x, y^{j}\right)$, the above inequality becomes

$$
\pi_{f}\left(x, y^{*, j}, y^{*,-j}\right)+\tilde{f}\left(x, y^{*, j}, y^{*,-j}\right) \leq \pi_{f}\left(x, y^{j}, y^{-j}\right)+\tilde{f}\left(x, y^{j}, y^{-j}\right), \forall y^{j} \in \mathcal{R}_{j}\left(x, y^{-j}\right) .
$$

Particularly for $y^{-j}=y^{*,-j}$ and $\forall y^{j} \in \mathcal{R}_{j}\left(x, y^{*,-j}\right)$, we have

$$
\pi_{f}\left(x, y^{*, j}, y^{*,-j}\right)+\tilde{f}\left(x, y^{*, j}, y^{*,-j}\right) \leq \pi_{f}\left(x, y^{j}, y^{*,-j}\right)+\tilde{f}\left(x, y^{j}, y^{*,-j}\right) .
$$


By rearranging the last inequality, we have an expression,

$$
\pi_{f}\left(x, y^{*, j}, y^{*,-j}\right)-\pi_{f}\left(x, y^{j}, y^{*,-j}\right)+\tilde{f}\left(x, y^{*, j}, y^{*,-j}\right) \leq \tilde{f}\left(x, y^{j}, y^{*,-j}\right), \forall y^{j} \in \mathcal{R}_{j}\left(x, y^{*,-j}\right) .
$$

Multiply both sides of (3.8) by $\delta_{j}(x)>0$ to get,

$$
\delta_{j}(x)\left[\pi_{f}\left(x, y^{*, j}, y^{*,-j}\right)-\pi_{f}\left(x, y^{j}, y^{*,-j}\right)\right]+\delta_{j}(x) \tilde{f}\left(x, y^{*, j}, y^{*,-j}\right) \leq \delta_{j}(x) \tilde{f}\left(x, y^{j}, y^{*,-j}\right) .
$$

Using the results from Lemma 3.4,

$$
\delta_{j}(x)\left[\pi_{f}\left(x, y^{*, j}\right)-\pi_{f}\left(x, y^{j}\right)\right]=\breve{f}_{i}\left(x, y^{*, j}, y^{*,-j}\right)-\breve{f}_{i}\left(x, y^{j}, y^{*,-j}\right) ;
$$

and using this in the last inequality, $\forall y^{j} \in \mathcal{R}_{j}\left(x, y^{*,-j}\right)$ we have

$$
\breve{f}_{j}\left(x, y^{*, j}, y^{*,-j}\right)-\breve{f}_{j}\left(x, y^{j}, y^{*,-j}\right)+\delta_{j}(x) \tilde{f}\left(x, y^{*, j}, y^{*,-j}\right) \leq \delta_{j}(x) \tilde{f}\left(x, y^{j}, y^{*,-j}\right) .
$$

Which implies, $\forall y^{j} \in \mathcal{R}_{j}\left(x, y^{*,-j}\right)$

$$
\breve{f}_{j}\left(x, y^{*, j}, y^{*,-j}\right)+\delta_{j}(x) \tilde{f}\left(x, y^{*, j}, y^{*,-j}\right) \leq \breve{f}_{j}\left(x, y^{j}, y^{*,-j}\right)+\delta_{j}(x) \tilde{f}\left(x, y^{j}, y^{*,-j}\right) .
$$

Or equivalently,

$$
f_{j}\left(x, y^{*, j}, y^{*,-j}\right) \leq f_{j}\left(x, y^{j}, y^{*,-j}\right), \forall y^{j} \in \mathcal{R}_{j}\left(x, y^{*,-j}\right) .
$$

From inequality (3.9) we can see that the tuple $y^{*}$, satisfies Definition 3.3. Therefore, $y^{*}$ is a global Nash reaction point of problem (3.3).

Under the assumptions (A1)-(A3), an equivalent reformulation of bilevel-MLMF game (3.1) as a bilevel game with single decision maker at both levels of the hierarchy is given by

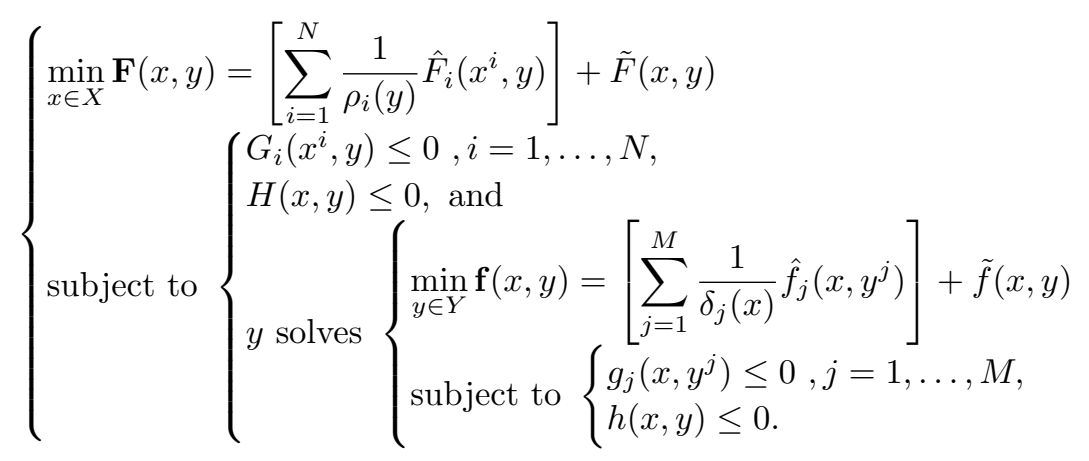

Proposition 3.7. Suppose that (3.1) satisfies the assumptions (A1)-(A3). If $\left(x^{*}, y^{*}\right)$ is a Stackelberg equilibrium point of (3.10), then $\left(x^{*}, y^{*}\right)$ is a Stackelberg-Nash equilibrium point of (3.1).

Proof. Follows from Theorems 3.5 and 3.6.

Note that when all the functions $\rho_{i}(y)$ and $\delta_{j}(x)$ are always equal to a constant 1, then problem (3.1) reduces to the quasi-potential game considered in [15]. Therefore, our result improves the one given in [15].

Remark 3.8. The idea described above can be extended to any finite $k$-level multi-leader multi-follower game by reformulating the problems on the same level with a single objective as stated above. That is, any multilevel multi-leader multi-follower game which satisfies assumptions (A1)-(A3) can be equivalently reformulated as a multilevel single-leader single-follower problem without increasing the vertical hierarchical levels. However the resulting multilevel optimization problem requires a solution approach that is different from the traditional Karush-Kuhn-Tucker (KKT) reformulation to avoid the effect of the complementarity conditions in the middle level problems. 


\section{Multi-PARAmetriC PROGRAmming FORMUlations AND MEthods}

A multi-parametric solution approach for multilevel optimization is a global solution strategy that works by rewriting the most inner level optimization problem as a multi-parametric problem, where the upper level optimization variables are considered as parameters. The resulting problem can be solved globally and the parametric solutions can be substituted into the most nearby upper level optimization problem. The key advantage of multi-parametric programming approach is that it provides a complete map of the optimal solution in the space of the varying parameters [20].

Multi-parametric programming techniques systematically subdivide the parameter space into characteristic regions where the optimal value and an optimizer are given as explicit functions of the parameters. A typical multi-parametric nonlinear program (mp-NLP) is generally defined as [5]:

$$
(\operatorname{NLP}(\theta)):\left\{\begin{array}{l}
\min _{x} f(x, \theta) \\
\text { s.t. }\left\{\begin{array}{l}
g_{i}(x, \theta) \leq 0, i=1, \ldots, p, \\
h_{j}(x, \theta)=0, j=1, \ldots, q,
\end{array}\right.
\end{array}\right.
$$

where $\theta \in \Theta \subseteq \mathbf{R}^{m}$ is the parameters vector, $x \in X \subseteq \mathbf{R}^{n}$ is the vector of the decision variables, and $f, g_{i}, h_{j}: \mathbf{R}^{n} \times \mathbf{R}^{m} \longrightarrow \mathbf{R}$ are parametric nonlinear functions.

The Lagrangian function associated with $(\operatorname{NLP}(\theta))$ is defined by:

$$
\mathcal{L}(x, \lambda, \mu, \theta)=f(x, \theta)+\sum_{i=1}^{p} \lambda_{i} g_{i}(x, \theta)+\sum_{j=1}^{q} \mu_{j}^{T} h_{j}(x, \theta),
$$

where $\lambda=\left(\lambda_{1}, \ldots, \lambda_{p}\right)^{T}$ and $\mu=\left(\mu_{1}, \ldots, \mu_{q}\right)^{T}$ are the Lagrange multiplier vectors associated with inequality and equality constraints $g_{i}$ and $h_{j}$, respectively.

If $\theta=\theta_{0}$ is fixed, we will have a classical nonlinear problem,

$$
\left(\operatorname{NLP}\left(\theta_{0}\right)\right):\left\{\begin{array}{l}
\min _{x} f\left(x, \theta_{0}\right) \\
\text { s.t. }\left\{\begin{array}{l}
g_{i}\left(x, \theta_{0}\right) \leq 0, i=1, \ldots, p, \\
h_{j}\left(x, \theta_{0}\right)=0, j=1, \ldots, q .
\end{array}\right.
\end{array}\right.
$$

The first-order sensitivity results for a parametric nonlinear programming problem $(\mathrm{NLP}(\theta))$ was presented by Fiacco [5], based on the following assumptions:

(C1) The functions defining problem $(\operatorname{NLP}(\theta))$ are twice continuously differentiable in $(x, \theta)$ in a neighborhood of $\left(x_{0}, \theta_{0}\right)$.

(C2) The second order sufficient conditions for a local minimum of problem $\left(\operatorname{NLP}\left(\theta_{0}\right)\right)$ hold at $x_{0}$ with associated Lagrange multipliers $\lambda_{0}$ and $\mu_{0}$.

(C3) The gradients $\nabla_{x} g_{i}\left(x_{0}, \theta_{0}\right)$, for all $i$ such that $g_{i}\left(x_{0}, \theta_{0}\right)=0$ and $\nabla_{x} h_{j}\left(x_{0}, \theta_{0}\right), j=1, \ldots, q$ are linearly independent.

(C4) Strict complementary slackness(SCS) holds at $\left(x_{0}, \theta_{0}\right)$, i.e. $\left(\lambda_{i}\right)_{0}>0$ for all $i$ such that $g_{i}\left(x_{0}, \theta_{0}\right)=0$.

Theorem 4.1 (Fiacco [5]). If assumptions (C1)-(C4) hold for problem $(N L P(\theta))$ at $\left(x_{0}, \theta_{0}\right)$, then:

(a) $x_{0}$ is a local isolated minimizing point of problem $\left(N L P\left(\theta_{0}\right)\right)$ and the associated multipliers $\lambda_{0}$ and $\mu_{0}$ are unique.

(b) For $\theta$ in a neighborhood of $\theta_{0}$, there exists a unique, once continuously differentiable vector function $z(\theta)=$ $[x(\theta), \lambda(\theta), \mu(\theta)]^{T}$ satisfying the second order sufficient conditions for a local minimum of problem $(N L P(\theta))$ such that $z\left(\theta_{0}\right)=z_{0}=\left[x_{0}, \lambda_{0}, \mu_{0}\right]^{T}$ and hence, $x(\theta)$ is a locally unique local minimum of problem $(N L P(\theta))$ with associated unique multipliers $\lambda(\theta)$ and $\mu(\theta)$. 
(c) For $\theta$ near $\theta_{0}$, the set of active inequalities is unchanged, strict complementary slackness holds for $\lambda_{i}(\theta)$ for $i$ such that $g_{i}(x(\theta), \theta)=0$, and the active constraint gradients are linearly independent at $x(\theta)$.

The derivative of $z(\theta)=[x(\theta), \lambda(\theta), \mu(\theta)]^{T}$ can be calculated near $\theta_{0}$ by the expression

$$
\nabla_{\theta} z(\theta)=[M(\theta)]^{-1} N(\theta),
$$

where $M(\theta)$ is the Jacobian with respect to $(x, \lambda, \mu)$ of the following Karush-Kuhn-Tucker system (satisfied by $z(\theta)$ near $\left.\theta=\theta_{0}\right)$ :

$$
\begin{aligned}
\nabla_{x} \mathcal{L}[x(\theta), \lambda(\theta), \mu(\theta), \theta] & =0 \\
\lambda_{i} g_{i}[x(\theta), \theta] & =0, \quad i=1, \ldots, p \\
h_{j}[x(\theta), \theta] & =0, \quad j=1, \ldots, q,
\end{aligned}
$$

and $N(\theta)$ is the negative of the Jacobian of the Karush-Kuhn-Tucker system with respect to $\theta$.

Corollary 4.2 (Fiacco [5]). Under the assumptions (C1)-(C4) a first-order estimation of the optimal solution $[x(\theta), \lambda(\theta), \mu(\theta)]$ in a neighborhood of $\theta_{0}$ is

$$
\left[\begin{array}{l}
x(\theta) \\
\lambda(\theta) \\
\mu(\theta)
\end{array}\right]=\left[\begin{array}{l}
x_{0} \\
\lambda_{0} \\
\mu_{0}
\end{array}\right]-M_{0}^{-1} \cdot N_{0} \cdot\left(\theta-\theta_{0}\right)+o(\|\theta\|),
$$

where $\left[x_{0}, \lambda_{0}, \mu_{0}\right]=\left[x\left(\theta_{0}\right), \lambda\left(\theta_{0}\right), \mu\left(\theta_{0}\right)\right], M_{0}=M\left(\theta_{0}\right), N_{0}=N\left(\theta_{0}\right)$, and $M(\theta)$ and $N(\theta)$ are defined as

$$
\begin{gathered}
M(\theta)=\left[\begin{array}{c|c|c}
\nabla_{x x}^{2} \mathcal{L} & \nabla_{x} g & \nabla_{x} h \\
\hline-\lambda \nabla_{x}^{T} g & \operatorname{diag}\left(-g_{i}\right) & 0 \\
\hline \nabla_{x}^{T} h & 0 & 0
\end{array}\right], \\
N(\theta)=\left[\nabla_{\theta x}^{2} \mathcal{L},-\lambda_{1} \nabla_{\theta}^{T} g_{1}, \ldots,-\lambda_{p} \nabla_{\theta}^{T} g_{p}, \nabla_{\theta}^{T} h_{1}, \ldots, \nabla_{\theta}^{T} h_{q}\right]^{T}
\end{gathered}
$$

and $\phi(\theta)=o(\|\theta\|)$ means that $\phi(\theta) /\|\theta\| \rightarrow 0$ as $\theta \rightarrow \theta_{0}$.

The assumptions (C1)-(C4) ensure that the inverse of $M_{0}$ exists and hence for problems involving convex $f, g$ and $h$, the parametric solutions within the corresponding critical regions, are necessary and sufficient.

The main question here is how to find a parametric solution $x(\theta)$ which remains stable in some subregion of the parameter space. We will consider this in the following two subsections.

\subsection{Multi-parametric problems with linear constraints}

Based on Corollary 4.2, Dua et al. [3] proposed an algorithm to solve (4.1) in the entire range of the varying parameters for general convex problems. The space of $\theta$ where solution (4.4) remains optimal to (4.1) is defined as the critical region, $\mathcal{C R}$, and can be obtained by using feasibility and optimality conditions [3]. Each piecewise linear approximation is confined to regions defined by feasibility and optimality conditions. If $\check{g}$ corresponds to the inactive polyhedral constraints and $\check{\lambda}$ to the Lagrangian multipliers of the active constraints, then the critical regions can be defined as,

$$
\mathcal{C R}= \begin{cases}\check{g}(x(\theta), \theta) \leq 0, & \text { Feasibility conditions, } \\ \check{\lambda}(\theta) \geq 0, & \text { Optimality conditions. }\end{cases}
$$

After defining the critical region $\mathcal{C R}$ on which the parametric solution is valid, if $\mathcal{C R}$ has not covered the parametric region, we repeat again the same mathematical procedure as in above with any new feasible parameter $\left(\theta=\theta_{0}\right)$ taken from the rest of parametric regions until the parametric region has been explored successfully as 
TABLE 1. Definition of the rest regions.

\begin{tabular}{lll}
\hline \hline Region & \multicolumn{2}{c}{ Inequalities } \\
\hline $\mathcal{C R}_{1}^{\text {Rest }}$ & $c_{1} \geq 0$, & $\theta_{1} \geq \theta_{2}^{L}, \theta_{2} \leq \theta_{2}^{U}$ \\
$\mathcal{C R}_{2}^{\text {Rest }}$ & $c_{1} \leq 0, c_{2} \geq 0$, & $\theta_{1} \geq \theta_{2}^{U}, \theta_{2} \leq \theta_{2}^{U}$ \\
$\mathcal{C R}_{3}^{\text {Rest }}$ & $c_{1} \leq 0, c_{2} \leq 0, c_{3} \geq 0$, & $\theta_{2}^{L} \leq \theta_{1} \geq \theta_{2}^{U}, \theta_{2}^{L} \leq \theta_{2}$ \\
\hline
\end{tabular}

described in [13]. To define the rest of the parametric region, consider $\mathcal{C} \mathcal{R}_{I}=\left[\theta^{L}, \theta^{U}\right]$ to be the overall parametric region (where $\theta^{L}$ and $\theta^{U}$ represent the lower and upper bounds of the parametric region) and let the inequalities, labeled by $c_{1} \leq 0, c_{2} \leq 0, c_{3} \leq 0$ define $\mathcal{C R}$. Now the rest of the parameter region $\mathcal{C R}^{\text {Rest }}=\mathcal{C R}_{I}-\mathcal{C} \mathcal{R}$ can be characterized by considering each of the inequalities which comprise $\mathcal{C} \mathcal{R}_{0}$, reversing their signs one by one and removing redundant constraints [4]. For example, consider inequality $c_{1} \leq 0$, the rest of the region can be addressed by reversing the sign of inequality $c_{1} \leq 0$ and removing redundant constraints in $\mathcal{C R}_{I}$, which is $\mathcal{C R}_{1}^{\text {Rest }}=c_{1} \geq 0, \theta_{1} \geq \theta_{2}^{L}, \theta_{2} \leq \theta_{2}^{U}$ where, $\theta=\left(\theta_{1}, \theta_{2}\right)$. Thus by considering the rest of the inequalities, the total of the rest region is given by, $\mathcal{C} \mathcal{R}^{\text {Rest }}=\mathcal{C R}_{1}^{\text {Rest }} \cup \mathcal{C R}_{2}^{\text {Rest }} \cup \mathcal{C R}_{3}^{\text {Rest }}$, where $\mathcal{C R}_{1}^{\text {Rest }}, \mathcal{C R}_{2}^{\text {Rest }}$ and $\mathcal{C R}_{3}^{\text {Rest }}$ are given in Table 1.

Finally, the optimal solution $x(\theta)$ can be expressed explicitly as a conditional piecewise linear function [3]:

$$
\left\{\begin{array}{ccc}
x=C_{1}+K_{1} \theta, & \text { if } & \theta \in \mathcal{C R}^{1} \\
x=C_{2}+K_{2} \theta, & \text { if } & \theta \in \mathcal{C R}^{2} \\
\vdots & & \vdots \\
x=C_{p}+K_{p} \theta, & \text { if } & \theta \in \mathcal{C R}^{p}
\end{array}\right.
$$

where $C_{i}$ are column vectors and $K_{i}$ are real matrices, whereas $\mathcal{C} \mathcal{R}^{i} \subseteq \mathbb{R}^{m}$ are critical regions and note that $\mathcal{C R}^{i}$ denotes the $i$ th critical region. For multi-parametric linear and quadratic problems, exact solutions can be computed using the first-order estimation.

\subsection{Multi-parametric problems with nonlinear constraints}

When a multi-parametric problem involves non-linear constraints, Fiacco [5] used penalty functions to estimate the first-order sensitivity results for a parametric nonlinear programming problem $(\mathrm{NLP}(\theta))$ by means of penalty function, define the following logarithmic-quadratic-barrier-penalty function for problem $(\mathrm{NLP}(\theta))$,

$$
W(x, \theta, t)=f(x, \theta)-t \sum_{i=1}^{p} \ln \left(-g_{i}(x, \theta)\right)+\frac{1}{2 t} \sum_{j=1}^{q} h_{j}^{2}(x, \theta) .
$$

Under assumptions $(\mathrm{C} 1)-(\mathrm{C} 4)$, and from penalty function theory (see Fiacco and McCormick [6]), we have the following results for problem $\left(\operatorname{NLP}\left(\theta_{0}\right)\right)$ :

(1) For $t>0$ and small, there exists a unique once continuously differentiable vector function $x\left(\theta_{0}, t\right)$ such that $x\left(\theta_{0}, t\right)$ is a locally unique minimizing point of $W\left(x, \theta_{0}, t\right)$ in

$$
K^{\#}\left(\theta_{0}\right)=\left\{x: g_{i}\left(x, \theta_{0}\right)<0, i=1, \ldots, p, \text { and } h_{j}\left(x, \theta_{0}\right)=0, j=1, \ldots, q\right\}
$$

and such that $x\left(\theta_{0}, t\right) \longrightarrow x\left(\theta_{0}, 0\right)=x^{*}$, and $x^{*}$ is a locally isolated minimizer of $\left(\operatorname{NLP}\left(\theta_{0}\right)\right)$.

(2) $\lim _{t \rightarrow 0} W\left(x\left(\theta_{0}, t\right), \theta_{0}, t\right)=f\left(x, \theta_{0}\right)$. 
Theorem 4.3 (Fiacco (1976) [5]). If assumptions (C1)-(C4) hold for $(N L P(\theta)$ ) then in a neighborhood about $(\theta, t)=\left(\theta_{0}, 0\right)$ there exists a unique once continuously differentiable vector function $z(\theta, t)=$ $[x(\theta, t), \lambda(\theta, t), \mu(\theta, t)]^{T}$ satisfying

$$
\begin{array}{rlrl}
\nabla_{x} \mathcal{L}[x, \lambda, \mu, \theta] & =0 & \\
\lambda_{i} g_{i}[x, \theta] & =t, \quad & i=1, \ldots, p \\
h_{j}[x(\theta), \theta] & =\mu_{j} t, \quad j=1, \ldots, q,
\end{array}
$$

with $z\left(\theta_{0}, 0\right)=\left(x^{*}, \lambda^{*}, \mu^{*}\right)^{T}$ and such that $(\theta, t)$ near $\left(\theta_{0}, 0\right)$ and $t>0, x(\theta, t)$ is a locally unique unconstrained local minimizing point of $W(x, \theta, t), g_{i}(x(\theta, t), \theta)<0, i=1, \ldots, m$, and $\nabla_{x}^{2} W(x(\theta, t), \theta, t)$ is positive definite.

The derivative of $z(\theta, t)=[x(\theta), \lambda(\theta), \mu(\theta)]^{T}$ can be calculated near $\left(\theta_{0}, 0\right)$ by the expression

$$
\nabla_{\theta} z(\theta, t)=[M(\theta, t)]^{-1} N(\theta, t),
$$

where $M$ and $-N$ are the Jacobians of the perturbed KKT system with respect to $(x, \lambda, \mu)$ and $\theta$, respectively.

Corollary 4.4 (Fiacco (1976) [5]). If assumptions (C1)-(C4) hold for $(N L P(\theta))$, then for any $\theta$ near $\theta_{0}$, then we have

(a) $\lim _{t \rightarrow 0^{+}} z(\theta, t)=z(\theta)$,

(b) $\lim _{t \rightarrow 0^{+}} \nabla_{\theta} z(\theta, t)=\nabla_{\theta} z(\theta)$.

Continuity of $f(x, \theta)$ and Corollary 4.4 imply that $\lim _{t \rightarrow 0^{+}} W^{*}(\theta, t)=f^{*}(\theta)$.

From the above arguments we can observe that whenever nonlinear convex constraints appear in the parametric problem (4.1), we apply the barrier method procedure to transform the nonlinear functional expression into the objective part and apply the procedure in Section 4.1 to obtain the required parametric solutions.

\section{Algorithm to solve multilevel-MLMF PROBlems Using EQUivalent REFORMULATION AND MULTI-PARAMETRIC APPROACH}

In this section we propose an appropriate solution approach to solve a classes of multilevel-MLMF games with a property that every objective function in the problem consists of separable terms and non-separable terms (but each of the non-separable terms can be written as a factor of two functions one of the factor being common across all players of the same level) and with non-degenerate polyhedral constraints in addition to possible convex non-linear ones. For the sake of clarity in presentation, the methodology is described using a general trilevel-MLMF game. However, the same approach can be extended to a general $k$-level case with appropriate adjustments.

\subsection{Equivalent reformulation of multilevel-MLMF games}

Consider a trilevel-MLMF game involving $N$ decision makers at the first-level, $M$ decision makers at the second-level and $L$ decision makers at the third-level which can be formulated mathematically as follows. For 
$n=1, \ldots, N$, the vector $\left(y_{1}^{n}, y_{2}, y_{3}\right)$ solves an optimization problem,

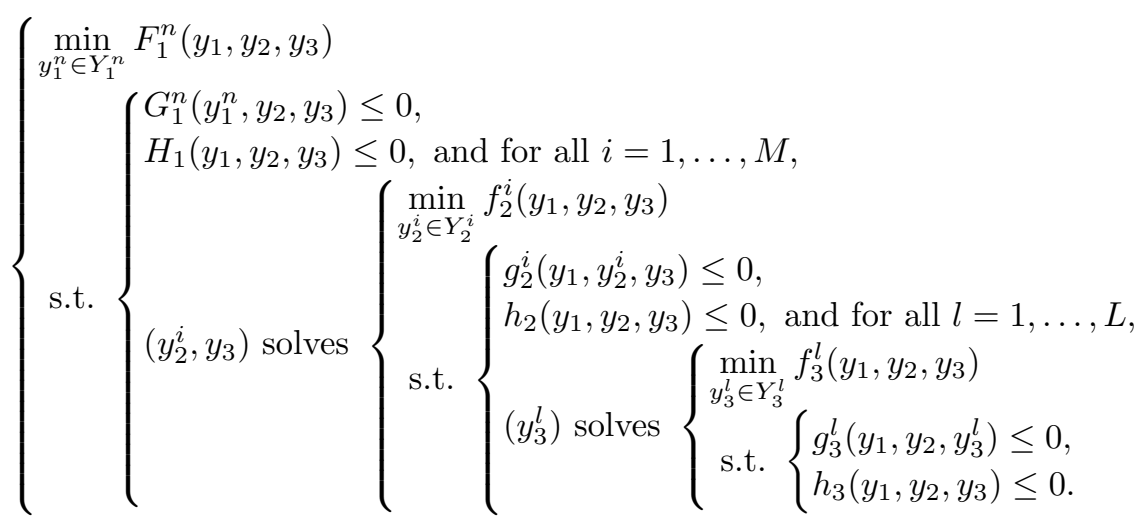

Assume that each of the objective functions of the third and second level followers is convex with respect to its own decision variable vector and the Guignard constraint qualifications [7] hold for the followers constraints.

We make the following assumptions on the structure of the objective functions of (5.1):

(B1) The objective functions at the first-level can be written as

$$
F_{1}^{n}\left(y_{1}, y_{2}, y_{3}\right)=\hat{F}_{1}^{n}\left(y_{1}^{n}, y_{2}, y_{3}\right)+\bar{F}_{1}^{n}\left(y_{1}^{-n}, y_{2}, y_{3}\right)+\rho_{1}^{n}\left(y_{2}, y_{3}\right) \tilde{F}_{1}\left(y_{1}, y_{2}, y_{3}\right),
$$

where $(\forall n) \quad\left(0<\rho_{1}^{n}\left(y_{2}, y_{3}\right)<\infty\right)$.

(B2) The objective functions at the second-level can be written as

$$
f_{2}^{i}\left(y_{1}, y_{2}, y_{3}\right)=\hat{f}_{2}^{i}\left(y_{1}, y_{2}^{i}, y_{3}\right)+\bar{f}_{2}^{i}\left(y_{1}, y_{2}^{-i}, y_{3}\right)+\rho_{2}^{i}\left(y_{1}, y_{3}\right) \tilde{f}_{2}\left(y_{1}, y_{2}, y_{3}\right),
$$

where $(\forall i) \quad\left(0<\rho_{2}^{i}\left(y_{1}, y_{3}\right)<\infty\right)$.

(B3) The objective functions at the third-level can be written as

$$
f_{3}^{j}\left(y_{1}, y_{2}, y_{3}\right)=\hat{f}_{3}^{j}\left(y_{1}, y_{2}, y_{3}^{j}\right)+\bar{f}_{3}^{j}\left(y_{1}, y_{2}, y_{3}^{-j}\right)+\rho_{3}^{j}\left(y_{1}, y_{2}\right) \tilde{f}_{3}\left(y_{1}, y_{2}, y_{3}\right),
$$

where $(\forall j) \quad\left(0<\rho_{3}^{j}\left(y_{1}, y_{2}\right)<\infty\right)$.

(B4) $\rho_{1}^{n}(\cdot), \rho_{2}^{i}(\cdot)$ and $\rho_{3}^{j}(\cdot)$ are twice continuously differentiable functions and uniformly bounded away from zero.

If (5.1) satisfies the assumptions (B1)-(B4), then it can be equivalently formulated as a trilevel optimization problem having a single decision maker at all levels as follows. The vector $\left(y_{1}, y_{2}, y_{3}\right)$ solves an optimization problem,

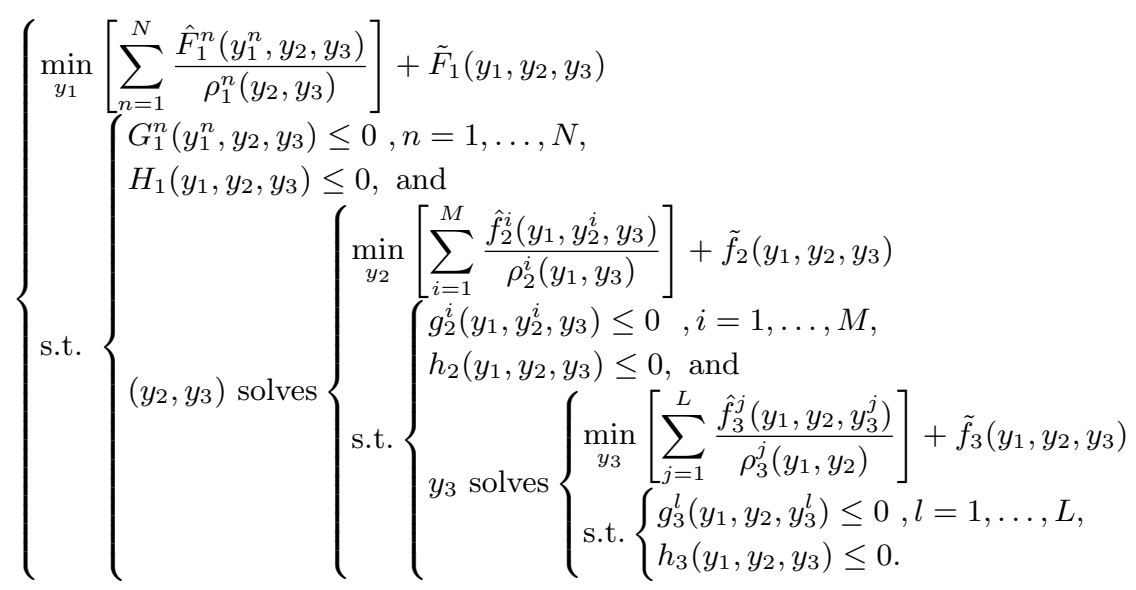

Then the following statement is a direct consequence of Proposition 3.7. Hence we state it here without proof. 
Proposition 5.1. Suppose that (5.1) satisfies the assumptions (B1)-(B4). If ( $\left.y_{1}^{*}, y_{2}^{*}, y_{3}^{*}\right)$ is a Stackelberg equilibrium point of (5.2), then $\left(y_{1}^{*}, y_{2}^{*}, y_{3}^{*}\right)$ is a Stackelberg-Nash equilibrium point of (5.1).

Note that the approach by Kulkarni and Shanbhag [15] leads to an MPEC for two level problems whose global solution provides an equilibrium to a bilevel-MLMF game. When we have more than two hierarchical levels, the nonconvex expression resulted from the lower-level problems makes it difficult to solve. For example, in tri-level programs the complementarity condition of the third-level KKT conditions makes the second-level problem nonconvex. Therefore, using the same transformation will result in a problem which is very difficult to solve (if it is tractable at all). To avoid this situation, we apply multi-parametric procedures for transforming the lower level problems. In this procedure, instead of embedding lower level problems into the middle level through the KKT conditions, we will equivalently transform the tri-level problem into a single-level problem by sequentially substituting the parametric solutions in problems of the middle and upper levels.

\subsection{Multi-parametric based algorithm to solve multilevel-MLMF}

The algorithm starts by reformulating the hierarchical multi-leader multi-follower problem as a multilevel optimization problem involving a single decision maker over the hierarchy as discussed in Sections 3 and 5.1. Then, each of the optimization problems in lower levels can be recast as multi-parametric programming problem where the variables from upper level problems are considered as parameters, and hence obtain an analytical parametric solution for the rational reaction set for each of the sub problems in the corresponding critical (stability) region of the parameter space. The basic steps of the proposed algorithm for tri-level MLMF problem (5.1) are described as follows.

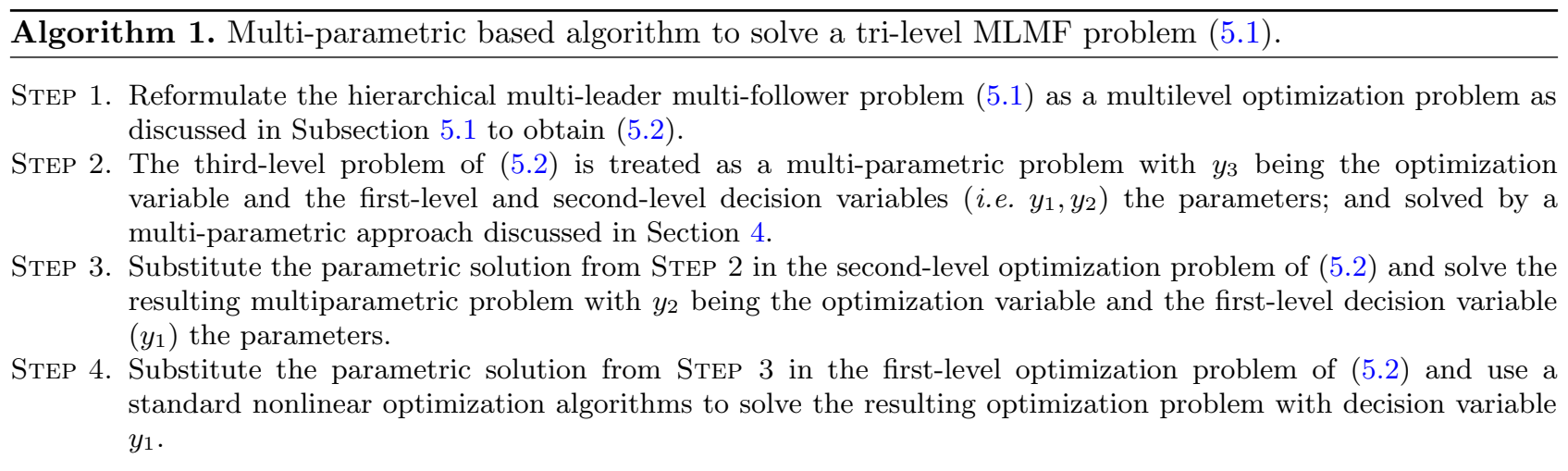

Remark 5.2. Since the number of partitions of the critical regions are finite as shown in [3,13], the algorithmic procedures described in Steps 2 and 3 terminate after a finite number of iterations. Hence, the above algorithm requires only a finite number of iterative procedures to arrive at the required solution.

Moreover, if all the involved functions are linear or quadratically convex, the solutions obtained through the above algorithm will be an exact global solution to the original problem.

\section{Illustrative Examples}

To apply the proposed solution algorithm, it is to be noted that each of the transformed problems at the lower levels must satisfy the required four conditions $(\mathrm{C} 1)-(\mathrm{C} 4)$. These conditions seem to be very strong and restrictive. However, many practical application problems satisfy these conditions. 
For example, the oligopoly market problem with divisible homogeneous products, that is described and analyzed in [11], satisfies all of the assumptions in our reformulation in Section 3. Therefore, it can be considered as a particular example of our proposed formulation.

The second example is the supply chain management problem. Supply chains are systems with multiple components such as supplier, manufacturer, distributer, retailer and customer, that exchange information with one another [2]. Mathematical models formulated to analyse the overall economic process of the supply chain management are usually described by MLMF games with at least three hierarchical levels (see for instance [9], where all the involved functions are assumed to be linear). Most of the deterministic versions of such problems satisfy the conditions required in our model formulation and solution algorithm.

Here below, we will illustrate the proposed method using selectively constructed numerical examples.

Example 6.1. Consider the following bilevel two-leader two-follower game:

$$
\begin{aligned}
& \left\{\begin{array}{l}
\min _{x_{1}} F_{1}(x, y)=-x_{1} y_{2}^{2}-x_{2} y_{1}^{2}+x_{1} e^{x_{2}} e^{y_{1}} \\
\min _{x_{2}} F_{2}(x, y)=x_{1}-3 x_{2} y_{1}+\left(y_{2}+1\right) x_{1} e^{x_{2}}
\end{array}\right. \\
& \text { s.t. }\left\{\begin{array}{l}
0 \leq x_{1}, x_{2} \leq 2, \\
\left\{\begin{array}{l}
\min _{y_{1}} f_{1}(x, y)=y_{1}^{2}+\left(1-x_{1}\right) y_{2}-\left(x_{2}^{2}+2\right) \ln \left(y_{1}+y_{2}+4\right), \\
\min _{2} f_{2}(x, y)=-y_{2}^{2}+\left(1-x_{2}\right) y_{1}-\left(x_{1}^{2}+3\right) \ln \left(y_{1}+y_{2}+4\right)
\end{array}\right. \\
\text { s.t. }\left\{\begin{array}{l}
x_{1}+2 y_{1}-y_{2}-2 \leq 0, \\
x_{1}-x_{2}-y_{1}+y_{2}-1 \leq 0, \\
0 \leq y_{1}, y_{2} \leq 2 .
\end{array}\right.
\end{array}\right.
\end{aligned}
$$

As it was discussed in Section 3 problem (6.1) can be equivalently reformulated as a bilevel single-leader singlefollower problem,

$$
\begin{aligned}
& \min _{x} F(x, y)=x_{1}\left(e^{x_{2}}-y_{2}^{2} e^{-y_{1}}\right)-\frac{3 y_{1} x_{2}}{y_{2}+1}
\end{aligned}
$$

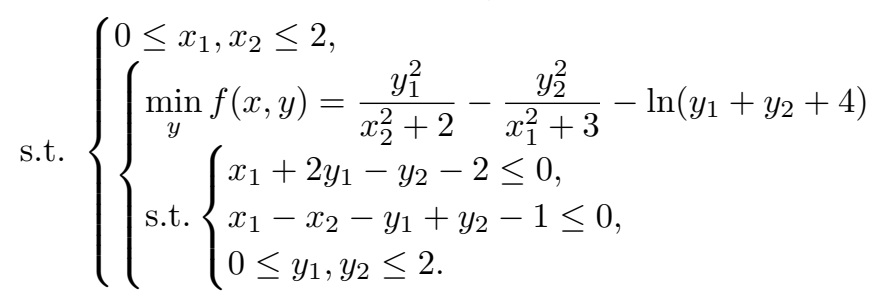

By treating the leaders decision variable, $x=\left(x_{1}, x_{2}\right)$ as a parameter, the inner problem in (6.2) can be considered as a multi-parametric problem:

$$
\begin{array}{rl}
\min _{y} & f(x, y)=\frac{y_{1}^{2}}{x_{2}^{2}+2}-\frac{y_{2}^{2}}{x_{1}^{2}+3}-\ln \left(y_{1}+y_{2}+4\right) \\
\text { s.t. }\left\{\begin{array}{l}
x_{1}+2 y_{1}-y_{2}-2 \leq 0 \\
x_{1}-x_{2}-y_{1}+y_{2}-1 \leq 0 \\
0 \leq y_{1}, y_{2} \leq 2
\end{array}\right.
\end{array}
$$

So we use multi-parametric approach discussed in Section 4 to solve (6.3). The Lagrangian of (6.3) is given by, $\mathcal{L}(x, y, \lambda)=f(x, y)+\lambda_{1} g_{1}(x, y)+\lambda_{2} g_{2}(x, y)$, where $g_{1}(x, y)=x_{1}+2 y_{1}-y_{2}-2$ and $g_{2}(x, y)=x_{1}-x_{2}-y_{1}+y_{2}-1$. 


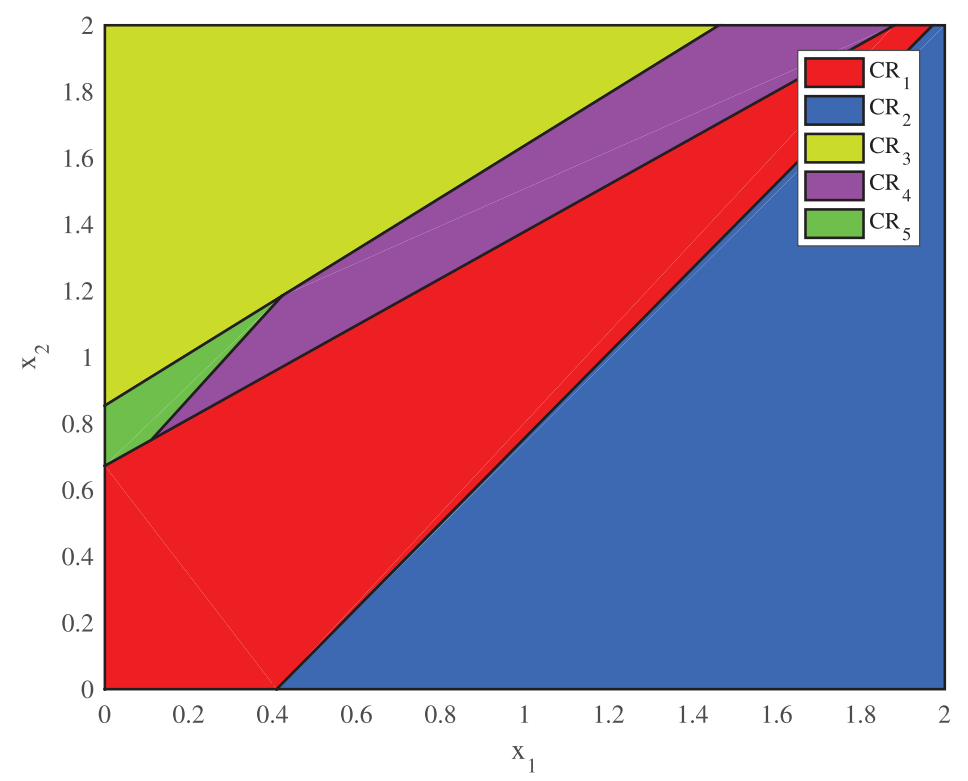

FiguRE 1. Critical regions for parametric problem (6.3).

$$
M(x)=\left[\begin{array}{c|c|c}
\nabla_{y y}^{2} \mathcal{L} & \nabla_{y} g_{1} & \nabla_{y} g_{2} \\
\hline-\lambda_{1} \nabla_{y}^{T} g_{1} & -g_{1} & 0 \\
\hline-\lambda_{2} \nabla_{y}^{T} g_{2} & 0 & -g_{2}
\end{array}\right] \text { and } N(x)=\left[\nabla_{x y}^{2} \mathcal{L},-\lambda_{1} \nabla_{x}^{T} g_{1},-\lambda_{2} \nabla_{x}^{T} g_{2}\right] .
$$

Then, $[y(x) \lambda(x) \mu(x)]^{T}=\left[\begin{array}{lll}y_{0} & \lambda_{0} & \mu_{0}\end{array}\right]^{T}-M_{0}^{-1} \cdot N_{0} \cdot\left(x-x_{0}\right)$ where $\left[y_{0} \lambda_{0} \mu_{0}\right]=\left[y\left(x_{0}\right) \lambda\left(x_{0}\right) \mu\left(x_{0}\right)\right], M_{0}=$ $M\left(x_{0}\right)$ and $N_{0}=N\left(x_{0}\right)$.

After exploring all the parameter spaces, the optimal solution to the problem (6.3) with their corresponding critical regions (see Fig. 1) are

$$
\begin{aligned}
& \mathcal{C R}_{1}=\left\{\begin{array}{l}
y^{*}(x)=\left[\begin{array}{l}
-3.8630 x_{1}+3.6393 x_{2}+1.2237 \\
-4.8630 x_{1}+4.6393 x_{2}+2.2237
\end{array}\right], \text { with } \\
0.7881 x_{1}-0.6156 x_{2} \leq 0.3224, \\
-0.5767 x_{1}+0.8170 x_{2} \leq 0.5498, \\
x_{1} \geq 0,0 \leq x_{2} \leq 2
\end{array}\right. \\
& \mathcal{C R}_{2}=\left\{\begin{array}{l}
y^{*}(x)=\left[\begin{array}{l}
-6.5148 x_{1}+4.5813 x_{2}+3.3640 \\
-7.5148 x_{1}+5.5813 x_{2}+4.3640
\end{array}\right], \text { with } \\
-0.7881 x_{1}+0.6156 x_{2} \leq-0.3224 \\
x_{1} \leq 2,0 \leq x_{2} \leq 2
\end{array}\right. \\
& \mathcal{C R}_{3}=\left\{\begin{array}{l}
y^{*}(x)=\left[\begin{array}{l}
0.2870 x_{2}-0.1114 \\
0.0107 x_{2}+1.9786
\end{array}\right], \text { with } \\
0.6167 x_{1}-0.7872 x_{2} \leq-0.6723, \\
x_{1} \geq 0, x_{2} \leq 2
\end{array}\right.
\end{aligned}
$$




$$
\begin{aligned}
& \mathcal{C R}_{4}=\left\{\begin{array}{l}
y^{*}(x)=\left[\begin{array}{l}
-14.7758 x_{1}+10.1658 x_{2}-2.4725 \\
-15.7758 x_{1}+11.1658 x_{2}-1.4725
\end{array}\right], \text { with } \\
-0.8125 x_{1}+0.5829 x_{2} \leq 0.3480, \\
-0.6167 x_{1}+0.7872 x_{2} \leq 0.6723, \\
0.5767 x_{1}-0.8170 x_{2} \leq-0.5498, \\
x_{2} \leq 2 ;
\end{array}\right. \\
& \text { and } \mathcal{C R}_{5}=\left\{\begin{array}{l}
y^{*}(x)=\left[\begin{array}{l}
-1.6379 x_{1}+1.0111 x_{2}+2.2657 \\
-2.6379 x_{1} 2.0111 x_{2}+3.2657
\end{array}\right], \text { with } \\
0.8125 x_{1}-0.5829 x_{2} \leq-0.3480 \\
-0.6167 x_{1}+0.7872 x_{2} \leq 0.6723 \\
0.5767 x_{1}-0.8170 x_{2} \leq-0.5498 \\
x_{1} \geq 0 .
\end{array}\right.
\end{aligned}
$$

This can be incorporated into the upper-level problem of (6.2) and the resulting nonlinear problems are solved in each critical regions. The following are the optimal solutions:In $\mathcal{C R}_{1},\left(x_{1}, x_{2}, y_{1}, y_{2}\right)=$ $(0.5157,0.4923,1.0234,2.0000)$ and objective value $F=-0.4014$.

In $\mathcal{C R}_{2}:\left(x_{1}, x_{2}, y_{1}, y_{2}\right)=(0.5035,0.1208,0.6372,1.2545)$ and objective value $F=0.0467$.

In $\mathcal{C R}_{3}:\left(x_{1}, x_{2}, y_{1}, y_{2}\right)=(0.2610,1.0586,0.1924,1.9899)$ and objective value $F=-0.3047$.

In $\mathcal{C R}_{4}:\left(x_{1}, x_{2}, y_{1}, y_{2}\right)=(0.5121,1.0345,0.4776,2.0000)$ and objective value $F=-0.3238$.

In $\mathcal{C R}_{5}:\left(x_{1}, x_{2}, y_{1}, y_{2}\right)=(0.3773,0.4820,2.1349,3.2395)$ and objective value $F=-0.5854$.

Now, comparing all the values of the objective of the leader in each of the critical regions, we can see that the objective value obtained in $\mathcal{C R}_{5}$ gives a better result. Hence we take $(0.3773,0.4820,2.1349,3.2395)$ as an optimal solution to the upper-problem of (6.2).

Therefore, the optimal solution to the bilevel multi-leader multi-follower problem (6.1) is $\left(x^{*}, y^{*}\right)=$ $(0.3773,0.4820,2.1349,3.2395)$ with optimal leaders objective $F_{1}=-0.9899$ and $F_{2}=-0.1191$; and optimal followers objective $f_{1}=1.5792$ and $f_{2}=-16.4213$.

Example 6.2. Consider the following nonlinear tri-level multi-follower programming problem:

$$
\begin{aligned}
& \left\{\begin{array}{l}
\min _{x_{1}} F_{1}^{1}(x, y, z)=\left(x_{1}-z_{1}\right)^{2}-\left(y_{1}+4\right) \cos \left(\frac{\pi}{2}\left(x_{2}-x_{1}\right)\right), \\
\min _{x_{2}} F_{1}^{2}(x, y, z)=\left(x_{2}-1\right)^{2}-\left(-y_{2}+z_{3}+3\right) \cos \left(\frac{\pi}{2}\left(x_{2}-x_{1}\right)\right)
\end{array}\right. \\
& \text { s.t. }\left\{\begin{array}{l}
0 \leq x_{1}, x_{2} \leq 1, \text { and } \\
\left\{\begin{array}{l}
\min _{y_{1}} f_{2}^{1}(x, y, z)=\left(y_{1}-1\right)^{2}+\left(y_{2}-2\right)^{2}+\left(x_{2}+z_{2}+5\right)\left(y_{1}^{2}+y_{2}\right)^{2}, \\
\min _{y_{2}} f_{2}^{2}(x, y, z)=-\frac{1}{2} y_{2}^{2}-y_{1} z_{1}+\left(x_{1}-z_{1}+6\right)\left(y_{1}^{2}+y_{2}\right)^{2},
\end{array}\right. \\
\text { s.t. }\left\{\begin{array}{l}
0 \leq y_{1}, y_{2} \leq 1, \text { and } \\
\left(\begin{array}{l}
\min _{z_{1}} f_{3}^{1}(x, y, z)=z_{1}^{2}+z_{1}+\left(y_{1}^{2}+1\right)\left(y_{2} x_{1}^{2}-z_{1}^{2}-z_{2}^{2}\right), \\
\min _{z_{2}} f_{3}^{2}(x, y, z)=z_{2}^{2}-y_{2} z_{2}+\left(2-y_{2}\right)\left(y_{2} x_{1}^{2}-z_{1}^{2}-z_{2}^{2}\right), \\
\min _{3} f_{3}^{3}(x, y, z)=-z_{3}^{2}+x_{2}\left(z_{1}-z_{2}\right)+\left(1+x_{2}\right)\left(y_{2} x_{1}^{2}-z_{1}^{2}-z_{2}^{2}\right),
\end{array}\right. \\
\text { s.t. } \begin{array}{l}
-y_{1}+2 y_{2}-2 z_{1}-z_{3}-2 \leq 0, \\
x_{1}-x_{2}+y_{1}-z_{2}+z_{3}-5 \leq 0, \\
4 x_{1}-2 y_{2}-z_{1}+z_{2}+z_{3}-3 \leq 0, \\
0 \leq z_{1}, z_{2}, z_{3} \leq 2 .
\end{array}
\end{array}\right.
\end{array}\right.
\end{aligned}
$$


Problem (6.4) is equivalently reformulated as tri-level problem having a single decision maker at each levels of the hierarchy:

$$
\begin{aligned}
& \min _{x} F_{1}(x, y, z)=\frac{\left(x_{1}-z_{1}\right)^{2}}{\left(y_{1}+4\right)}+\frac{\left(x_{2}-1\right)^{2}}{\left(y_{2}-z_{3}+3\right)}-\cos \left(\frac{\pi}{2}\left(y_{2}-z_{1}\right)\right) \\
& \text { s.t. }\left\{\begin{array}{l}
0 \leq x_{1}, x_{2} \leq 1, \text { and } \\
\min _{y} f_{2}(x, y, z)=\frac{\left(y_{1}-1\right)^{2}}{\left(x_{2}+z_{2}+5\right)}-\frac{y_{2}^{2}}{2\left(x_{1}-z_{1}+6\right)}+\left(y_{1}^{2}+y_{2}\right)^{2},
\end{array}\right. \\
& \text { s.t. }\left\{\begin{array}{l}
0 \leq y_{1}, y_{2} \leq 1, \text { and } \\
\min _{z} f_{3}(x, y, z)=\frac{z_{1}^{2}+z_{1}}{y_{1}^{2}+1}+\frac{z_{2}^{2}-y_{2} z_{2}}{2-y_{2}}-\frac{z_{3}^{2}}{x_{2}+y_{1}+1}+y_{2} x_{1}^{2}-z_{1}^{2}-z_{2}^{2}, \\
\text { s.t. }\left\{\begin{array}{l}
-y_{1}+2 y_{2}-2 z_{1}-z_{3}-2 \leq 0, \\
x_{1}-x_{2}+y_{1}-z_{2}+z_{3}-5 \leq 0, \\
4 x_{1}-2 y_{2}-z_{1}+z_{2}+z_{3}-3 \leq 0 \\
0 \leq z_{1}, z_{2}, z_{3} \leq 2 .
\end{array}\right.
\end{array}\right.
\end{aligned}
$$

By treating the first and second level decision variables, $(x, y)$ as parameters, the third-level problem in (6.5) can be considered as a multi-parametric problem (with the parameter vector $(x, y)$ ):

$$
\left\{\begin{array}{l}
\min _{z} f_{3}(x, y, z)=\frac{z_{1}^{2}+z_{1}}{y_{1}^{2}+1}+\frac{z_{2}^{2}-y_{2} z_{2}}{2-y_{2}}-\frac{z_{3}^{2}}{x_{2}+y_{1}+1}+y_{2} x_{1}^{2}-z_{1}^{2}-z_{2}^{2} \\
\text { s.t. }\left\{\begin{array}{l}
-y_{1}+2 y_{2}-2 z_{1}-z_{3}-2 \leq 0 \\
x_{1}-x_{2}+y_{1}-z_{2}+z_{3}-5 \leq 0 \\
4 x_{1}-2 y_{2}-z_{1}+z_{2}+z_{3}-3 \leq 0 \\
0 \leq z_{1}, z_{2}, z_{3} \leq 2 .
\end{array}\right.
\end{array}\right.
$$

The Lagrangian of (6.6) is given by $\mathcal{L}(x, y, z, \lambda)=f_{3}(x, y, z)+\lambda_{1} g_{1}+\lambda_{2} g_{2}+\lambda_{3} g_{3}$, where $g_{1}=-y_{1}+2 y_{2}-2 z_{1}-$ $z_{3}-2, g_{2}=x_{1}-x_{2}+y_{1}-z_{2}+z_{3}-5$ and $g_{3}=4 x_{1}-2 y_{2}-z_{1}+z_{2}+z_{3}-3$. After exploring all the parameter spaces, the optimal solution to the problem (6.6) with their corresponding critical regions (see Fig. 2) are:

$$
\mathcal{C R}_{1}=\left\{\begin{array}{l}
z^{*}(x, y)=\left[\begin{array}{l}
-0.7111 y_{1}-0.9028 y_{2}+1.7361 x_{1}-0.0611 \\
-0.3556 y_{1}+0.7361 y_{2}-1.5694 x_{1}+2.5944 \\
-0.3556 y_{1}+0.3611 y_{2}-0.6944 x_{1}+0.3444
\end{array}\right], \text { with } \\
0.1731 y_{1}+0.7667 y_{2}-0.6183 x_{1}-0.4946 \leq 0 \\
0 \leq y_{1}, y_{2} \leq 1,0 \leq x_{1}, x_{2} \leq 1
\end{array}\right.
$$

$$
\text { and } \mathcal{C R}_{2}=\left\{\begin{array}{l}
z^{*}(x, y)=\left[\begin{array}{c}
-1.2255 y_{1}+0.7353 \\
1.6071 y_{2}-2.8571 x_{1}+1.2857 \\
0 \\
0.1731 y_{1}-0.7667 y_{2}+0.6183 x_{1}+0.4946 \leq 0 \\
0 \leq y_{1}, y_{2} \leq 1,0 \leq x_{1}, x_{2} \leq 1
\end{array}\right] \text {, with } \\
-0 .
\end{array}\right.
$$

Using these solutions into the second-level problem of (6.5) results in a multi-parametric problems of parameter $x$. Again by employing a multi-parametric approach, in $\mathcal{C R}_{1}$ we have,

$$
\left\{\begin{array}{l}
y^{*}(x)=\left[\begin{array}{c}
0.0561 x_{1}-0.0358 x_{2}+0.3433 \\
-0.0424 x_{1}+0.0270 x_{2}+0.0077
\end{array}\right], \text { with } \\
0 \leq x_{1}, x_{2} \leq 1
\end{array}\right.
$$




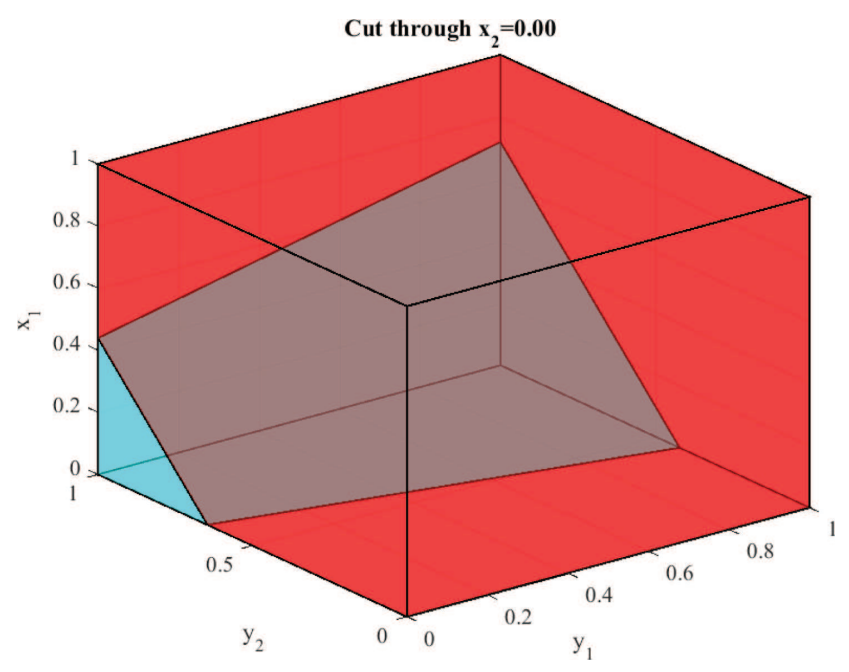

FiguRE 2. Critical regions for parametric problem (6.3).

and in $\mathcal{C R}_{2}$ we have,

$$
\left\{\begin{array}{l}
y^{*}(x)=\left[\begin{array}{c}
-0.0838 x_{1}-0.0105 x_{2}+0.1864 \\
0.8254 x_{1}+0.0024 x_{2}+0.6030
\end{array}\right], \text { with } \\
0 \leq x_{1}, x_{2} \leq 1
\end{array}\right.
$$

Incorporating these solutions into the leader problem of (6.5) and solving the resulting problem, we obtain: In $\mathcal{C R}_{1},(x, y, z)=(0.3230,0.4720,0.3446,0.0068,0.2486,1.9699,0)$ and objective value $F_{1}=-0.8782$. In $\mathcal{C R}_{2}$, $(x, y, z)=(0.4792,0.6299,0.1397,1.0000,0.5641,1.5237,0)$ and objective value $F_{1}=-0.9022$. Since the objective value obtained in $\mathcal{C R}_{2}$ is better we can take it as an optimal solution to the upper-problem of (6.5).

Therefore, the optimal solution to the tri-level multi-leader multi-follower problem (6.4) is $\left(x^{*}, y^{*}, z^{*}\right)=$ $(0.4792,0.6299,0.1397,1.0000,0.5641,1.5237,0)$ with optimal leaders objective $F_{1}^{1}=-4.8533$ and $F_{1}^{2}=$ -1.8072 ; optimal second-level objectives $f_{2}^{1}=9.1757$ and $f_{2}^{2}=5.0693$; and optimal third-level objectives $f_{3}^{1}=-1.5750, f_{3}^{2}=-1.6124$ and $f_{3}^{3}=-4.5332$.

Example 6.3. Consider the following bilevel two-leader two-follower problem with nonlinear constraints:

$$
\begin{aligned}
& \left\{\begin{array}{l}
\min _{x_{1}} F_{1}(x, y)=x_{1}^{2}+x_{2} y_{1}+y_{2}^{2}+x_{1} x_{2}, \\
\min _{x_{2}} F_{2}(x, y)=x_{2}^{2}-4 x_{2}+x_{1}^{2}-y_{1}+x_{1} x_{2}
\end{array}\right.
\end{aligned}
$$

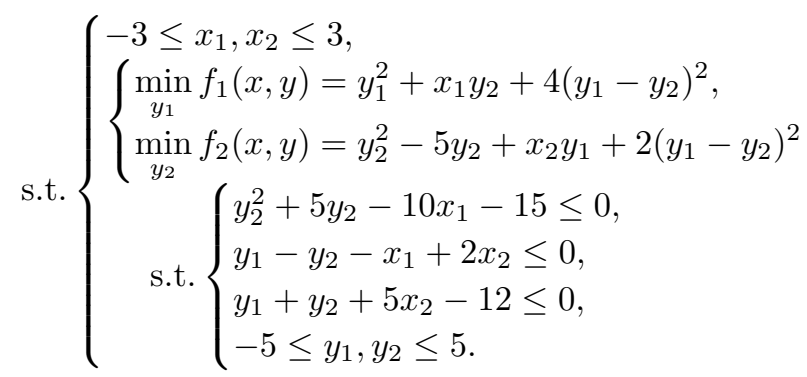


Problem (6.7) can be equivalently reformulated as a bilevel single-leader single-follower problem,

$$
\begin{aligned}
& \min _{x} F(x, y)=x_{1}^{2}+x_{2} y_{1}-4 x_{2}+x_{1} x_{2}
\end{aligned}
$$

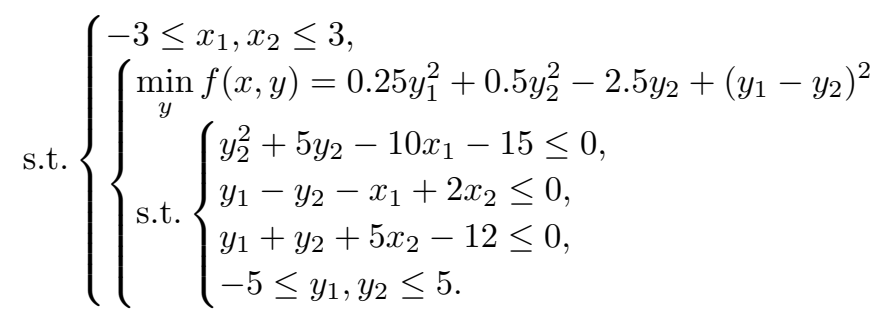

By treating the leaders decision variable, $x=\left(x_{1}, x_{2}\right)$ as a parameter, the inner problem in (6.8) can be considered as a multi-parametric problem:

$$
\begin{gathered}
\min _{y} f(x, y)=0.25 y_{1}^{2}+0.5 y_{2}^{2}-2.5 y_{2}+\left(y_{1}-y_{2}\right)^{2} \\
\text { s.t. }\left\{\begin{array}{l}
y_{2}^{2}+5 y_{2}-10 x_{1}-15 \leq 0 \\
y_{1}-y_{2}-x_{1}+2 x_{2} \leq 0 \\
y_{1}+y_{2}+5 x_{2}-12 \leq 0 \\
-5 \leq y_{1}, y_{2} \leq 5
\end{array}\right.
\end{gathered}
$$

So we use multi-parametric approach discussed in Section 4 to solve (6.9). Here, $G(x, y)=y_{2}^{2}+5 y_{2}-10 x_{1}-15$ and $g(x, y)=\left[y_{1}-y_{2}-x_{1}+2 x_{2}, y_{1}+y_{2}+5 x_{2}-12\right]$. So for $x \in X^{*}$ the barrier function, $\psi(x, y)$, is define as $\psi(x, y)=-\ln (-G(x, y))$ with domain $\{y: G(x, y)<0\}$.

Assuming the intersection of the domain of the logarithmic barrier and the polyhedral sets is nonempty, i.e., $Y_{B}(x)=\left\{y: G(x, y)<0, g(x, y) \leq 0,-3 \leq x_{1}, x_{2} \leq 3,-5 \leq y_{1}, y_{2} \leq 5\right\} \neq \emptyset$, and for $t>0$ we can define the barrier approximation for (6.9) as

$$
\begin{aligned}
W^{*}(x, t)=\min _{y}\left\{0.25 y_{1}^{2}+0.5 y_{2}^{2}-2.5 y_{2}+\left(y_{1}-y_{2}\right)^{2}+t \psi(x, y)\right\} \\
\text { s.t. }\left\{\begin{array}{l}
y_{1}-y_{2}-x_{1}+2 x_{2} \leq 0 \\
y_{1}+y_{2}+5 x_{2}-12 \leq 0 \\
-5 \leq y_{1}, y_{2} \leq 5 .
\end{array}\right.
\end{aligned}
$$

Problem (6.10) is a MPP with parameter $x=\left(x_{1}, x_{2}\right)$ and its Lagrangian becomes,

$$
\begin{gathered}
\mathcal{L}(x, y, \lambda, t)=0.25 y_{1}^{2}+0.5 y_{2}^{2}-2.5 y_{2}+\left(y_{1}-y_{2}\right)^{2}+t \psi(x, y)+\lambda^{T} g(x, y), \\
M(x, t)=\left[\begin{array}{c|c}
\nabla_{y y}^{2} \mathcal{L} & \nabla_{y} g \\
\hline-\lambda \nabla_{y}^{T} g & \operatorname{diag}(-g)
\end{array}\right], N(x, t)=\left[\nabla_{x y}^{2} \mathcal{L},-\lambda \nabla_{x} g\right]^{T}, \\
\\
{\left[\begin{array}{c}
y(x, t) \\
\lambda(x, t)
\end{array}\right]=\left[\begin{array}{c}
y_{0} \\
\lambda_{0}
\end{array}\right]-\left[M_{0}(t)\right]^{-1} \cdot N_{0}(t) \cdot\left[x-x_{0}\right],}
\end{gathered}
$$

where $\left(y_{0}, \lambda_{0}\right)=\left(y\left(x_{0}\right), \lambda\left(x_{0}\right)\right), M_{0}(t)=M\left(x_{0}, t\right)$ and $N_{0}(t)=N\left(x_{0}, t\right)$.

By solving the barrier problem (6.10), as $t \rightarrow 0^{+}$, the solutions and the critical regions will be,

$$
y(x)= \begin{cases}{\left[\begin{array}{c}
0.6681 x_{1}-1.3340 x_{2}+1.6663 \\
-0.3319 x_{1}+0.6660 x_{2}+1.6663
\end{array}\right]} & \text { on } \mathcal{C R}_{1} \\
{\left[\begin{array}{c}
0.5 x_{1}-3.5 x_{2}+6 \\
-0.5 x_{1}-1.5 x_{2}+6
\end{array}\right]} & \text { on } \mathcal{C R}_{2}\end{cases}
$$




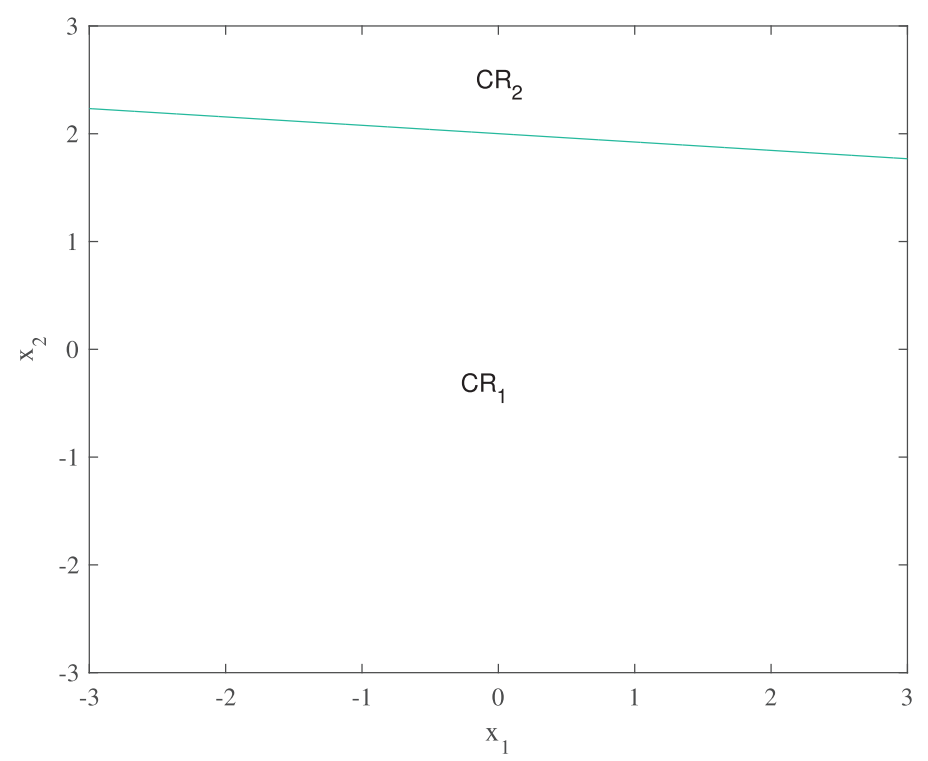

Figure 3. Critical regions for the problem (6.10).

$$
\begin{aligned}
\mathcal{C R}_{1} & =\left\{\begin{array}{l}
-0.0774 x_{1}+0.9970 x_{2}-1.9948 \leq 0, \\
-3 \leq x_{1} \leq 3, x_{2} \geq-3
\end{array}\right. \\
\mathcal{C R}_{2} & =\left\{\begin{array}{l}
0.0774 x_{1}-0.9970 x_{2}+1.9948 \leq 0 \\
-3 \leq x_{1} \leq 3, x_{2} \leq 3
\end{array}\right.
\end{aligned}
$$

where $\mathcal{C} \mathcal{R}_{1}$ and $\mathcal{C} \mathcal{R}_{2}$ are as shown in Figure 3.

Then, the critical regions of (6.9) is determined as follows:

$$
\begin{aligned}
& \mathcal{C R}^{L}=\{\mathcal{C R}\} \cap\{x: G(y(x), x) \leq 0\}, \\
& y_{L}(x)= \begin{cases}{\left[\begin{array}{c}
0.6681 x_{1}-1.3340 x_{2}+1.6663 \\
-0.3319 x_{1}+0.6660 x_{2}+1.6663
\end{array}\right]} & \text { on } \mathcal{C R}_{1}^{L} \\
{\left[\begin{array}{c}
0.5 x_{1}-3.5 x_{2}+6 \\
-0.5 x_{1}-1.5 x_{2}+6
\end{array}\right]} & \text { on } \mathcal{C R}_{2}^{L}\end{cases} \\
& \mathcal{C R}_{1}^{L}=\left\{\begin{array}{l}
0.0774 x_{1}+0.9970 x_{2}-1.9948 \leq 0, \\
0.1102 x_{1}^{2}-0.4421 x_{1} x_{2}-12.7660 x_{1}+0.4435 x_{2}^{2}+5.5491 x_{2}-3.8917 \leq 0, \\
-3 \leq x_{1} \leq 3, x_{2} \geq-3 ;
\end{array}\right. \\
& \mathcal{C R}_{2}^{L}=\left\{\begin{array}{l}
-0.0774 x_{1}-0.9970 x_{2}+1.9948 \leq 0, \\
0.25 x_{1}^{2}+1.5 x_{1} x_{2}-18.5 x_{1}+2.25 x_{2}^{2}-25.5 x_{2}+51 \leq 0, \\
-3 \leq x_{1} \leq 3, x_{2} \leq 3,
\end{array}\right.
\end{aligned}
$$

where $\mathcal{C} \mathcal{R}_{1}^{L}$ and $\mathcal{C} \mathcal{R}_{2}^{L}$ are as shown in Figure 4 .

The rest of the spaces will be,

$$
\mathcal{C R}_{1}^{N L}=\left\{\begin{array}{l}
0.0774 x_{1}+0.9970 x_{2}-1.9948 \leq 0 \\
0.1102 x_{1}^{2}-0.4421 x_{1} x_{2}-12.7660 x_{1}+0.4435 x_{2}^{2}+5.5491 x_{2}-3.8917 \geq 0 \\
-3 \leq x_{1} \leq 3, x_{2} \geq-3
\end{array}\right.
$$




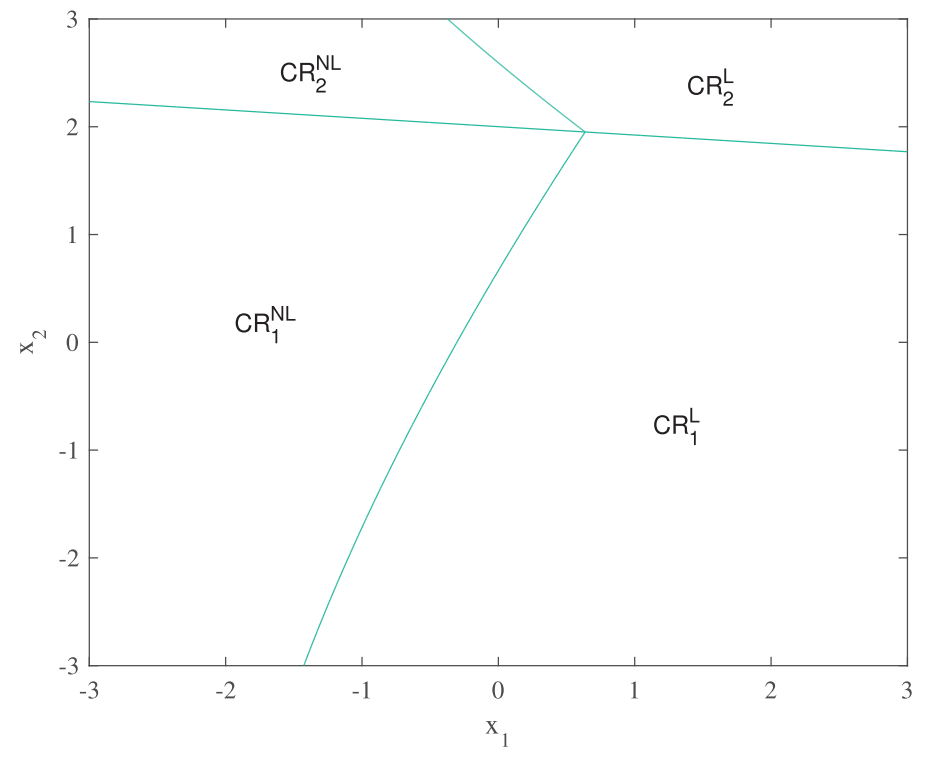

Figure 4. Critical regions for the problem (6.10).

$$
\mathcal{C} \mathcal{R}_{2}^{N L}=\left\{\begin{array}{l}
-0.0774 x_{1}-0.9970 x_{2}+1.9948 \leq 0 \\
0.25 x_{1}^{2}+1.5 x_{1} x_{2}-18.5 x_{1}+2.25 x_{2}^{2}-25.5 x_{2}+51 \geq 0 \\
-3 \leq x_{1} \leq 3, x_{2} \leq 3
\end{array}\right.
$$

Next we will determine the nonlinear solution as follows:

In $\mathcal{C} \mathcal{R}_{1}^{N L}$ the solution is determined from $Y_{N L}(x)=\arg \left\{G(x, y)=0, g_{2}(x, y)<0\right\}$

$$
y_{N L}(x)=\arg \left\{y_{2}^{2}+5 y_{2}-10 x_{1}-15=0, y_{1}+y_{2}+5 x_{2}-12<0\right\},
$$

which is infeasible.

In $\mathcal{C R}_{2}^{N L}$ the solution is determined from $Y_{N L}(x)=\arg \left\{G(x, y)=0, g_{2}(x, y)=0\right\}$

$$
y_{N L}(x)=\arg \left\{y_{2}^{2}+5 y_{2}-10 x_{1}-15=0, y_{1}+y_{2}+5 x_{2}-12=0\right\},
$$

which results in

$$
Y_{N L}(x)=\left[\begin{array}{c}
\mp 0.5 \sqrt{5\left(8 x_{1}+17\right)}-5 x_{2}+14.5 \\
\pm 0.5 \sqrt{5\left(8 x_{1}+17\right)}-2.5
\end{array}\right]
$$

Since $y=(-0.5120,2.7547)$ at $x=(0.6362,1.9515)$ which is a common point for $\mathcal{C R}_{1}^{L}, \mathcal{C} \mathcal{R}_{2}^{L}$ and $\mathcal{C} \mathcal{R}_{2}^{L}$, the only nonlinear parametric solution is

$$
Y_{N L}(x)=\left[\begin{array}{c}
-0.5 \sqrt{5\left(8 x_{1}+17\right)}-5 x_{2}+14.5 \\
0.5 \sqrt{5\left(8 x_{1}+17\right)}-2.5
\end{array}\right]
$$

which is valid in $\mathcal{C R}_{2}^{N L}$. 
Therefore, the optimal solution is given by,

$$
y(x)= \begin{cases}{\left[\begin{array}{c}
0.6681 x_{1}-1.3340 x_{2}+1.6663 \\
-0.3319 x_{1}+0.6660 x_{2}+1.6663
\end{array}\right]} & \text { on } \mathcal{C R}_{1}^{L} \\
{\left[\begin{array}{c}
0.5 x_{1}-3.5 x_{2}+6 \\
-0.5 x_{1}-1.5 x_{2}+6
\end{array}\right]} & \text { on } \mathcal{C R}_{2}^{L} \\
\text { Infeasible } & \text { on } \mathcal{C R}_{1}^{N L} \\
{\left[\begin{array}{c}
-0.5 \sqrt{5\left(8 x_{1}+17\right)}-5 x_{2}+14.5 \\
0.5 \sqrt{5\left(8 x_{1}+17\right)}-2.5
\end{array}\right]} & \text { on } \mathcal{C R}_{2}^{N L}\end{cases}
$$

where $\mathcal{C} \mathcal{R}_{1}^{L}, \mathcal{C} \mathcal{R}_{2}^{L}, \mathcal{C} \mathcal{R}_{1}^{N L}$ and $\mathcal{C} \mathcal{R}_{2}^{N L}$ are as indicated in Figure 4 .

Incorporating the solution $y(x)$ into the upper-level problem of (6.8) and solving the resulting nonlinear problems in each critical regions, we get following optimal solutions:

In $\mathcal{C R}_{1}^{L},\left(x_{1}, x_{2}, y_{1}, y_{2}\right)=(-0.4365,1.0305,-0.0000,2.4975)$ and objective value $F=-2.2574$.

In $\mathcal{C} \mathcal{R}_{2}^{L}:\left(x_{1}, x_{2}, y_{1}, y_{2}\right)=(1.2998,1.9000,-0.0000,2.5002)$ and objective value $F=3.7788$.

In $\mathcal{C R}_{2}^{N L}:\left(x_{1}, x_{2}, y_{1}, y_{2}\right)=(-0.5710,1.1428,4.8437,1.4421)$ and objective value $F=-2.2857$.

Now, comparing all the values of the objective of the leader in each of the critical regions, we can see that the objective value obtained in $\mathcal{C} \mathcal{R}_{2}^{N L}$ gives a better result. Hence we take the solution in $\mathcal{C R}_{2}^{N L}$ as an optimal solution to the upper-problem of (6.8).

Therefore, the optimal solution to the bilevel multi-leader multi-follower problem $(6.7)$ is $\left(x^{*}, y^{*}\right)=$ $(-0.5710,1.1428,4.8437,1.4421)$ with optimal leaders objective $F_{1}=7.2880$ and $F_{2}=-8.4355$; and optimal followers objective $f_{1}=68.9219$ and $f_{2}=41.1532$.

\section{Conclusion}

This work proposes a solution procedure for a class of hierarchical multi-leader multi-follower games whose objective functions at each level have non-separable terms and some polyhedral constraints. The solution procedure transforms the given problem into an equivalent multilevel hierarchical problems having single decision maker at each level of the hierarchy and without increasing the level of hierarchy in the problem. The equivalent reformulation maintains the equilibrium points of the original problem, so that we can use any of the existing methods to solve the resulting multilevel problem with one player at each level. In this article the multi-parametric approach is employed to solve the resulting multilevel optimization problem. The proposed equivalence reformulation does not require the smoothness of the involved functions. However, due to the requirements of the multi-parametric solution methods (that is used to solve multilevel hierarchical problems in this article), we additionally imposed second order smoothness conditions as well as convexity of the lower level problems. These conditions may not be necessary if one uses other methods (like the heuristic methods) to solve the resulting equivalent multilevel hierarchical optimization problems.

Acknowledgements. The research of the first author was supported by the International Science Program (ISP) of Sweden, through a research project at the Department of Mathematics, Addis Ababa University.

\section{REFERENCES}

[1] J. Bracken and J. McGill, Mathematical programs with optimization problems in the constraints. Oper. Res. 21 (1973) 37-44.

[2] B. Brunaud, I.E. Grossmann, Perspectives in multilevel decision-making in the process industry. Front. Eng. Manag. 4 (2017) 256-270.

[3] V. Dua, N.A. Bozinis and E.N. Pistikopoulos, A multiparametric programming approach for mixed-integer quadratic engineering problems. Comput. Chem. Eng. 26 (2002) 715-733. 
[4] V. Dua and E.N. Pistikopoulos, An algorithm for the solution of multiparametric mixed integer linear programming problems. Ann. Oper. Res. 99 (2000) 123-139.

[5] A.V. Fiacco, Sensitivity analysis for nonlinear programming using penalty methods. Math. Program. 10 (1976) $287-311$.

[6] Fiacco, A.V., McCormick, G.P.: Nonlinear Programming: Sequential Unconstrained Minimization Techniques. J. Wiley and Sons, Inc., New York, London, Sydney, Toronto (1968)

[7] M. Guignard, Generalized Kuhn-Tucker conditions for mathematical programming problems in a Banach space. SIAM J. Cont. 7 (1969) 232-241.

[8] J. Han, G. Zhang, Y. Hu, J. Lu, Solving tri-level programming problems using a particle swarm optimization algorithm. In: The 10th IEEE Conference on Industrial Electronics and Applications (2015) 569-574.

[9] J. Han, G. Zhang, J. Lu, Y. Hu and S. Ma, Model and algorithm for multi-follower tri-level hierarchical decision-making, edited by C. Loo, K. Yap, K. Wong, A. Beng-Jin, K. Huang. In: Neural Information Processing. ICONIP 2014, Part III. Lecture Notes in Computer Science, Vol. 8836. Springer, Switzerland (2014) 398-406.

[10] M. Hu and M. Fukushima, Multi-leader-follower games: models, methods and applications. J. Oper. Res. Soc. Jpn 58 (2015) $1-23$.

[11] L.A. Julien, On noncooperative oligopoly equilibrium in the multiple leader-follower game. Eur. J. Oper. Res. 256 (2017) $650-662$

[12] S.M. Kassa, Three-level global resource allocation model for HIV control: A hierarchical decision system approach. Math. Biosci. Eng. 15 (2018) 255-273.

[13] A.M. Kassa and S.M. Kassa, Approximate solution algorithm for multi-parametric non-convex programming problems with polyhedral constraints. Int. J. Optim. Control: Theor. Appl. 4 (2014) 89-98.

[14] A.M. Kassa, S.M. Kassa, Deterministic solution approach for some classes of nonlinear multilevel programs with multiple follower. J. Global Optim. 68 (2017) 729-747.

[15] A.A. Kulkarni, U.V. Shanbhag, An existence result for hierarchical Stackelberg v/s Stackelberg games. IEEE Trans. Automat. Contr. 60 (2015) 3379-3384.

[16] S. Leyffer, T. Munson, Solving multi-leader-common-follower games. Optim. Methods Softw. 25 (2010) $601-623$.

[17] K. Okuguchi, Expectations and stability in oligopoly models. In: Lecture Notes in Economics and Mathematical Systems, vol. 138. Springer-Verlag, Berlin (1976)

[18] J.S. Pang, M. Fukushima, Quasi-variational inequalities, generalized Nash equilibria, and multi-leader-follower games. Comput. Manage. Sci. 2 (2005) 21-56.

[19] J.S. Pang, M. Fukushima, Quasi-variational inequalities, generalized Nash equilibria, and multi-leader-follower games. Comput. Manage. Sci. 6 (2009) 373-375.

[20] E. Pistikopoulos, M. Georgiadis, V. Dua, Multi-parametric programming: theory, algorithms and applications. Wiley-VCH Verlag GmbH \& Co. KGaA (2007).

[21] H.D. Sherali, A multiple leader Stackelberg model and analysis. Oper. Res. 32 (1984) 390-404

$[22]$ C.L. Su, Analysis on the forward market equilibrium model. Oper. Res. Lett. 35 (2007) 74-82.

[23] L. Sun, Equivalent bilevel programming form for the generalized Nash equilibrium problem. J. Math. Res. 2 (2010) 8-13.

[24] Q. Wang, F. Yang, Y. Liu, Bilevel programs with multiple followers. J. Syst. Sci. Complex. 13 (2000) $265-276$.

[25] A.B. Zewde, S.M. Kassa, A method for solving some class of multilevel multi-leader multi-follower programming problems, edited by H.A. Le-Thi, H.M. Le, T. Pham-Dinh. In: Optimization of Complex Systems: Theory, Models, Algorithms and Applications. WCGO 2019. Advances in Intelligent Systems and Computing, Vol. 991. Springer Nature, Switzerland (2020) $589-599$.

[26] A.B. Zewde, S.M. Kassa, Multi-parametric approach for multilevel multi-leader-multi-follower games using equivalent reformulations. J. Math. Comput. Sci. 11 (2021) 2955-2980.

\section{Subscribe to Open (S2O)}

\section{A fair and sustainable open access model}

This journal is currently published in open access under a Subscribe-to-Open model (S2O). S2O is a transformative model that aims to move subscription journals to open access. Open access is the free, immediate, online availability of research articles combined with the rights to use these articles fully in the digital environment. We are thankful to our subscribers and sponsors for making it possible to publish this journal in open access, free of charge for authors.

\section{Please help to maintain this journal in open access!}

Check that your library subscribes to the journal, or make a personal donation to the $\mathrm{S} 2 \mathrm{O}$ programme, by contacting subscribers@edpsciences.org

More information, including a list of sponsors and a financial transparency report, available at: https://www. edpsciences.org/en/maths-s2o-programme 